\title{
Charged scalar production at the compact linear collider for the $S_{\mathbf{3}} \otimes \mathbb{Z}_{2}$ model
}

\author{
G. De Conto ${ }^{1, \mathrm{a}}$, A. C. B. Machado ${ }^{1, \mathrm{~b}}$, J. Montaño ${ }^{2, \mathrm{c}}$, P. Chimenti ${ }^{3, \mathrm{~d}}$ \\ ${ }^{1}$ Centro de Ciências Naturais e Humanas, Universidade Federal do ABC, Santo André, SP 09210-580, Brazil \\ 2 CONACYT-Facultad de Ciencias Físico Matemáticas, Universidad Michoacana de San Nicolás de Hidalgo, 58060 Morelia, Michoacán, Mexico \\ ${ }^{3}$ Departamento de Física, Centro de Ciências Exatas, Universidade Estadual de Londrina, Londrina, PR 86051-990, Brazil
}

Received: 10 February 2020 / Accepted: 29 October 2020 / Published online: 8 November 2020

(C) The Author(s) 2020

\begin{abstract}
We present a model with $S_{3} \otimes \mathbb{Z}_{2}$ model plus a sterile neutrino and its phenomenological expectations for the production of charged scalars at the compact linear collider. At tree level, our model predicts a total cross section in between 0.1 and $10^{-5} \mathrm{pb}$ for the $e^{-} e^{+} \rightarrow H^{+} H^{-}$process, considering all possible mass values for the charged scalar in the CLIC experiment. We also show that this prediction holds regardless of the masses of the other exotic particles and their couplings. We also show that an indirect detection from its effects in the $e \bar{e} \rightarrow e \bar{e}+$ missing energy process is possible under specific conditions, or a direct detection under other circumstances. However, one cannot use this process to study the sterile neutrinos present in this model, given that they have a small influence in the total cross-section for the direct detection of the exotic scalar to be possible.
\end{abstract}

\section{Introduction}

The Standard Model (SM) of fundamental interactions is still an incomplete theory, even though it was shown as an experimentally proven theory with a high predictive power, once we have observed dark matter (DM) from galaxy rotation curves, and there is no viable candidate for DM in the SM as it is today, the SM has an unknown missing piece. We also have the fact that neutrinos in the SM are non-massive, therefore, to have massive neutrinos, the only requirement is the addition of right handed (or sterile neutrino) fields to the SM matter content. However, because neutrinos are only charged under weak isopsin and hyperchagre, some consequences arise because of the introduction of their right -

\footnotetext{
a e-mail: george.de.conto@gmail.com (corresponding author)

be-mail: a.c.b.machado1@gmail.com

ce-mail: jmontano@conacyt.mx

de-mail: pietro.chimenti@uel.br
}

handed components, for example, neutrinos can be a majorana particles. This means that neutrinos are the most elusive of the known particles. Their weak interactions make it very difficult to study their properties, but, this may be seen as a good quality for the neutrinos, once they can hold the key to solve several mysteries in particle physics and cosmology. For instance, the simplest dark matter candidate, at least from the point of view of particle physics, is the neutrino.

The DM particle candidates may have very different masses (for reviews of DM candidates see e.g. [1-4]): massive gravitons with mass $\sim 10^{-19} \mathrm{eV}$ [5], axions with mass $\sim 10^{-6} \mathrm{eV}[6]$, sterile neutrinos having mass in the $\mathrm{keV}$ range [7], sypersymmetric (SUSY) particles (gravitinos [8], neutralinos [9], axinos [10] with their masses ranging from $\mathrm{eV}$ to hundreds $\mathrm{GeV}$, supersymmetric Q-balls [11], WIMPZILLAs with the mass $\sim 10^{13} \mathrm{GeV}[12,13]$, and many others). Thus, the mass of DM particles becomes an important characteristic which may help to distinguish between various DM candidates and, more importantly, may help to differentiate among different models beyond the SM.

Sterile neutrinos as Dark Matter candidates, with the mass in the $\mathrm{keV}$ range, were originally suggested in [7]. Because these particles are neutral with respect to all SM charges, they have to be massive and, while unstable, they can have a lifetime longer than the age of the Universe (controlled by the active-sterile mixing parameter). If they exist, they were produced at high temperatures in the early Universe. The sterile neutrinos are never in thermal equilibrium, unlike other cosmic relic particles (e.g. photons, neutrinos or hypothetical WIMPs) because of their feeble interaction, so their exact production mechanism is model-dependent. In Ref. [14] they discuss the most important observational constraints on these particles as DM candidates.

Despite being a candidate for DM known for more than 15 years [7], recently the sterile neutrinos once again stood out as it was shown in Ref. [15]. If three sterile right-handed neu- 
trinos are added to the SM, it is possible to explain simultaneously the data on neutrino oscillations (see e.g. [16-18] for a review) and the Dark Matter in the Universe, without introducing any new physics above electro-weak scale. Moreover, if the masses of two of these particles are between $\sim 100 \mathrm{MeV}$ and the electro-weak scale, and are almost degenerate, it is also possible to generate the correct baryon asymmetry of the Universe (see e.g. $[15,19,20]$ ). The third (lightest) sterile neutrino can have a mass in the $\mathrm{keV}-\mathrm{MeV}$ range and be coupled to the rest of the matter weakly enough to provide a viable (cold or warm) DM candidate.

In this context, we have proposed an extension of the model with a $S_{3} \otimes \mathbb{Z}_{2}$ symmetry [21,22] assuming that righthanded neutrinos can be sterile. The model consists of two scalar doublets and two scalar singlets plus the SM particles, with their potential having the most general lagrangian allowed by the chosen symmetry. We present a full analysis of the scalar potential of the model, showing the scalar mass eigenstates, and identify the SM Higgs boson among these by imposing that its Yukawa couplings are the same in our model and in the SM.

Models with a $S_{3}$ symmetry have been studied in a variety of previous works. Among these, some are: the model's scalar potential, including its mass eigenstates and self-couplings [23-25], the quark sector of these models [26], and the neutrino masses and their mixing [27]. Despite some of these topics overlapping our work, we feel that what we present here is relevant. In our model two scalar singlets are added, which further increases the scalar sector and its complexity, leading to different mass eigenstates and Yukawa sectors. Therefore, our results are not the same as the ones shown in the works just cited.

Also, the extension of the scalar sector brings new charged scalars, which in turn provide new sources of $\mathrm{CP}$ violation, given that they come up in vertices with the form $\bar{f}_{L} f_{R} H^{ \pm}$, where $f_{L, R}$ denotes left and right-handed fermions. While in the SM we have vertices with the form $\bar{f}_{L} f_{L} W^{ \pm}$that give rise to the CKM matrix, the $\bar{f}_{L} f_{R} H^{ \pm}$vertices mixes left and right-handed fermions, bringing in new combinations of matrices that diagonalize the fermion mass eigenstates. These new combinations of unitary matrices generate new sources of $\mathrm{CP}$ violation, which are necessary to solve other problems faced by the SM such as the observed matter-antimatter asymmetry of the Universe [28-30] and mass differences in mesonic systems [31,32].

In order to explore the properties and phenomenological consequences of both type of particles from our model, charged scalars and sterile neutrinos, we are going to study the tree level process $e^{-} e^{+} \rightarrow H^{+} H^{-}$, where the sterile neutrinos participate as virtual particles and the charged scalars as final state particles. Because we are studying electronpositron collisions, we considered the compact linear collider (CLIC), a multi TeV linear electron-positron collider under development $[33,34]$, to calculate the cross section for the $e^{-} e^{+} \rightarrow H^{+} H^{-}$process.

CLIC is a TeV-scale high-luminosity linear $e^{+} e^{-}$collider under development by international collaborations hosted by CERN. For an optimal exploitation of its physics potential it is foreseen to be built and operated in stages at center-ofmass energies of $380 \mathrm{GeV}, 1.5 \mathrm{TeV}$ and $3 \mathrm{TeV}$, for a site length ranging between $11 \mathrm{~km}$ and $50 \mathrm{~km}$. The high collision energy combined with the large luminosity and clean environment of the $e^{+} e^{-}$collisions will enable to measure the properties of SM particles, such as the Higgs boson and the top quark, with unparalleled precision. CLIC might also discover indirect effects of very heavy new physics by probing the parameters of the Standard Model Effective Field Theory with an unprecedented level of precision. This includes new particles detected in non-standard signatures, such as electrically charged scalars. The construction of the first CLIC energy stage could start as early as 2026 and the first beams would be available by 2035, marking the beginning of a physics programme spanning 25-30 years and providing excellent sensitivity to Beyond Standard Model physics, through direct searches and via a broad set of precision measurements of SM processes, particularly in the Higgs and top-quark sectors.

The outline of the paper is as follows: first we present the model and its Lagrangian, then we focus into its scalar sector calculating the mass eigenstates and indentify the SM Higgs boson within those, and finally calculate the cross-section for the $e^{-} e^{+} \rightarrow H^{+} H^{-}$process in our model, followed by our conclusions.

\section{The $S_{3} \otimes \mathbb{Z}_{2}$ model plus a sterile neutrino}

Here we will use the $S_{3}$ discrete symmetry in order to obtain a model with 3 Higgs doublets, being two of them inert. The $S_{3}$ symmetry consists of all permutations among three objects. However, the representation of order 3 is reducible and is decomposed in two irreducible representations: $\mathbf{3}=$ $\mathbf{1} \oplus \mathbf{2}$. Here we will write only the multiplications involving two doublets and two singlets (which will be used here for obtaining the Yukawa interactions) and the scalar potential that is invariant under the full symmetry, $S U(2)_{L} \otimes U(1)_{Y} \otimes$ $S_{3} \otimes \mathbb{Z}_{2}$. Let $\left[x_{1}, x_{2}\right]$ and $\left[y_{1}, y_{2}\right]$ be two doublets of $S_{3}$, the multiplication $\mathbf{2} \otimes \mathbf{2}$ is given by

$$
\begin{aligned}
{\left[\begin{array}{l}
x_{1} \\
x_{2}
\end{array}\right]_{2} \otimes\left[\begin{array}{l}
y_{1} \\
y_{2}
\end{array}\right]_{2}=} & {\left[x_{1} y_{1}+x_{2} y_{2}\right]_{1}+\left[x_{1} y_{2}-x_{2} y_{1}\right]_{1^{\prime}} } \\
& +\left[\begin{array}{l}
x_{1} y_{2}+x_{1} y_{2} \\
x_{1} y_{1}-x_{2} y_{2}
\end{array}\right]_{2^{\prime}}=\mathbf{1} \oplus \mathbf{1}^{\prime} \oplus \mathbf{2}^{\prime}
\end{aligned}
$$

being $\mathbf{1}$ and $\mathbf{1}^{\prime}$ singlets and $\mathbf{2}^{\prime}$ a doublet. Besides we have that $\mathbf{1} \otimes \mathbf{1}=\mathbf{1}$ and $\mathbf{1}^{\prime} \otimes \mathbf{1}^{\prime}=\mathbf{1}$. For more details about this and other discrete symmetries see Ref. [35]. The $Z_{N}$ group, that 
is Abelian, can be represented as discrete rotations, whose generators corresponds to a $2 \pi / N$ rotation.

The Yukawa interactions are given by

$$
\begin{aligned}
-\mathcal{L}_{\text {Yukawa }}^{\text {leptons }}= & G_{i j}^{l} \bar{L}_{i} l_{j R} S+G_{i j}^{v} L_{i} v_{j L} S+G_{i s}^{N} L_{i} \in N_{s R} S \\
& \left.+\frac{y_{d}}{2}\left[\overline{N_{d R}^{c}} N_{d R}\right]_{2} \zeta_{d}\right]_{1}+\frac{y_{s d}}{2}\left[\overline{N_{s R}^{c}}\left[N_{d R} \zeta_{d}\right]_{2}\right]_{1} \\
& +\frac{F_{i s}^{v}}{\Lambda} \bar{L}^{c}{ }_{i} N_{S}\left[D_{d} \zeta_{d}^{*}\right]_{1}+\text { H.c. }
\end{aligned}
$$

where $i, j=e, \mu, \tau$ and $d=1,2$. In Eq. $2 L$ is the SM lepton doublet, $l$ is the SM charged lepton, $v$ is the SM neutrino, $D_{1,2}$ and $S$ are scalar SU(2) doublets, $N_{s}$ and $N_{1,2}$ are righthanded neutrinos. $G^{l}, G^{v}, G^{N}, y_{d}, y_{s d}$ and $F^{v}$ are matrices for the Yukawa couplings and $\Lambda$ is an energy scale above the electroweak scale for the dimension 5 operator. For more details about the model see [22], the difference in our work is that we changed the interactions of $N_{s}$ to make it lighter than the other right-handed neutrinos.

The scalar $\mathrm{SU}(2)$ doublets, $D_{1,2}$ and $S$, are introduced to generate Majorana masses for the left-handed neutrinos, according to the scotogenic model. These doublets may also be candidates for dark matter, a possibility that was studied in [22]. In order to have a lighter neutrino than the scale of the heavier Majorana neutrinos we use the $S_{3}$ symmetry, that separates the right-hand neutrinos into a doublet and singlet (this lighter neutrino may fit the evidences of lighter sterile neutrinos). However, for the symmetry to be conserved it is necessary to introduce two scalar singlets $\left(\zeta_{1,2}\right)$, so that the heavier right-handed neutrinos have Majorana masses on the $\mathrm{TeV}$ scale.

\section{Scalar sector}

The scalar sector of the model is presented as follows:

$$
\begin{aligned}
S & =\left(\begin{array}{c}
S^{+} \\
\frac{1}{\sqrt{2}}\left(v_{S M}+\operatorname{Re} S^{0}+i \operatorname{Im} S^{0}\right)
\end{array}\right), \\
D_{1,2} & =\left(\begin{array}{c}
D_{1,2}^{+} \\
\frac{1}{\sqrt{2}}\left(\eta_{1,2}+i \chi_{1,2}\right) .
\end{array}\right),
\end{aligned}
$$

plus two real the singlets $\zeta_{i}=\frac{v_{i}+\xi_{i}}{\sqrt{2}}, i=1,2$.

The scalar potential invariant under the gauge and $S_{3} \otimes \mathbb{Z}_{2}$ symmetries is

$$
\begin{aligned}
V_{S_{3}}= & \mu_{s}^{2} S^{\dagger} S+\mu_{d}^{2}\left[D^{\dagger} \otimes D\right]_{1}+\mu_{\zeta}^{2}\left[\zeta_{d} \otimes \zeta_{d}\right]_{1} \\
& +\mu_{12}^{2} \zeta_{1} \zeta_{2}+a_{1}\left(\left[D^{\dagger} \otimes D\right]_{1}\right)^{2}+a_{2}\left[\left[D^{\dagger} \otimes D\right]_{1^{\prime}}\left[D^{\dagger} \otimes D\right]_{1^{\prime}}\right] \\
& +a_{3}\left[\left(D^{\dagger} \otimes D\right)_{2^{\prime}}\left(D^{\dagger} \otimes D\right)_{2^{\prime}}\right]_{1} \\
& +a_{4}\left(S^{\dagger} S\right)^{2}+a_{5}\left[D^{\dagger} \otimes D\right]_{1} S^{\dagger} S+a_{6}\left[\left[S^{\dagger} D\right]_{2^{\prime}}\left[S^{\dagger} D\right]_{2^{\prime}}\right]_{1}
\end{aligned}
$$

$$
\begin{aligned}
& + \text { H.c. }]+a_{7} S^{\dagger}\left[D \otimes D^{\dagger}\right]_{1} S+b_{1} S^{\dagger} S\left[\zeta_{d} \otimes \zeta_{d}\right]_{1} \\
& +b_{2}\left[D^{\dagger} \otimes D\right]_{1}\left[\zeta_{d} \otimes \zeta_{d}\right]_{1}+b_{3}\left[\left[D^{\dagger} \otimes D\right]_{2^{\prime}}\left[\zeta_{d} \otimes \zeta_{d}\right]_{2^{\prime}}\right]_{1} \\
& +b_{4}\left[\left[\left[D^{\dagger} \otimes D\right]_{1^{\prime}}\left[\zeta_{d} \otimes \zeta_{d}\right]_{1^{\prime}}\right]_{1}+c_{1}\left(\left[\zeta_{d} \otimes \zeta_{d}\right]_{1}\right)^{2}\right. \\
& +c_{2}\left[\left[\zeta_{d} \otimes \zeta_{d}\right]_{2^{\prime}}\left[\zeta_{d} \otimes \zeta_{d}\right]_{2^{2}}\right]_{1},
\end{aligned}
$$

with $\mu_{d}^{2}>0$ since $\left\langle D_{1,2}^{0}\right\rangle=0$ is guaranteed by the $S_{3}$ symmetry. The parameter $a_{6}$ has been chosen real without loss of generality.

We can write Eq. (4) explicitly as

$$
V\left(S, D, \zeta_{d}\right)=V^{(2)}+V^{(4 a)}+V^{(4 b)}+V^{(4 c)},
$$

where

$$
\begin{aligned}
V^{(2)}= & \mu_{S M^{2}}^{2} S^{\dagger} S+\mu_{d}^{2}\left(D_{1}^{\dagger} D_{1}+D_{2}^{\dagger} D_{2}\right)+\mu_{\zeta}^{2}\left(\zeta_{1}^{2}+\zeta_{2}^{2}\right) \\
& +\mu_{12}^{2} \zeta_{1} \zeta_{2}, \\
V^{(4 a)}= & a_{1}\left(D_{1}^{\dagger} D_{1}+D_{2}^{\dagger} D_{2}\right)^{2}+a_{2}\left(D_{1}^{\dagger} D_{2}-D_{2}^{\dagger} D_{1}\right)^{2} \\
& +a_{3}\left[\left(D_{1}^{\dagger} D_{2}+D_{2}^{\dagger} D_{1}\right)^{2}+\left(D_{1}^{\dagger} D_{1}-D_{2}^{\dagger} D_{2}\right)^{2}\right] \\
& +a_{4}\left(S^{\dagger} S\right)^{2}+a_{5}\left(D_{1}^{\dagger} D_{1}+D_{2}^{\dagger} D_{2}\right) S^{\dagger} S \\
& +a_{6}\left[\left(S^{\dagger} D_{1}\right)^{2}+\left(S^{\dagger} D_{2}\right)^{2}+H . c .\right] \\
& +a_{7}\left[\left(S^{\dagger} D_{1}\right)\left(D_{1}^{\dagger} S\right)+\left(S^{\dagger} D_{2}\right)\left(D_{2}^{\dagger} S\right)\right], \\
V^{(4 b)}= & b_{1} S^{\dagger} S\left(\zeta_{1}^{2}+\zeta_{2}^{2}\right)+b_{2}\left(D_{1}^{\dagger} D_{1}+D_{2}^{\dagger} D_{2}\right)\left(\zeta_{1}^{2}+\zeta_{2}^{2}\right) \\
& +b_{3}\left[\left(D_{1}^{\dagger} D_{2}+D_{2}^{\dagger} D_{1}\right)\left(\zeta_{1} \zeta_{2}+\zeta_{1} \zeta_{2}\right)\right. \\
& \left.+\left(D_{1}^{\dagger} D_{1}-D_{2}^{\dagger} D_{2}\right)\left(\zeta_{1}^{2}-\zeta_{2}^{2}\right)+H . c .\right] \\
& +b_{4}\left[\left(D_{1}^{\dagger} D_{2}-D_{2}^{\dagger} D_{1}\right)\left(\zeta_{1} \zeta_{2}-\zeta_{1} \zeta_{2}\right)\right] \\
V^{(4 c)}= & c_{1}\left(\zeta_{1}^{2}+\zeta_{2}^{2}\right)^{2}+c_{2}\left[\left(\zeta_{1} \zeta_{2}+\zeta_{2} \zeta_{1}\right)^{2}+\left(\zeta_{1}^{2}-\zeta_{2}^{2}\right)^{2}\right],
\end{aligned}
$$

where we have used $\left[\zeta_{d} \zeta_{d}\right]_{2^{\prime}}=\left(\zeta_{1} \zeta_{2}+\zeta_{2} \zeta_{1}, \zeta_{1} \zeta_{1}-\zeta_{2} \zeta_{2}\right)$. Here, we will consider all the couplings to be real parameters i.e., there is no $C P$ violation in the scalar sector. The $S_{3}$ symmetry forbids linear terms with the doublets $D_{1}, D_{2}$ in the scalar potential and also some of the Yukawa interactions with charged leptons. This ensures the inert character of the these doublets after the $S_{3}$ symmetry is introduced. Notice that, although the term $\mu_{12}^{2}$ breaks softly the $S_{3}$ symmetry, it happens in the sector of the singlets $\zeta_{1,2}$ and does not spoil the inert character of the doublets.

From Eq. (4), we obtain the following stability conditions for the potential (i.e., setting its derivatives to zero):

$$
\begin{aligned}
& \frac{1}{2} v_{S M}\left(2 a_{4} v_{S M}^{2}+b_{1}\left(v_{1}^{2}+v_{2}^{2}\right)+2 \mu_{S M}^{2}\right)=0, \\
& \frac{1}{2}\left(b_{1} v_{1} v_{S M}^{2}+2 v_{1}\left(c_{1}+c_{2}\right)\left(v_{1}^{2}+v_{2}^{2}\right)+\mu_{12}^{2} v_{2}\right)+\mu_{\zeta}^{2} v_{1}=0, \\
& \frac{1}{2} b_{1} v_{2} v_{S M}^{2}+v_{2}\left(c_{1}+c_{2}\right)\left(v_{1}^{2}+v_{2}^{2}\right)+\frac{\mu_{12}^{2} v_{1}}{2}+\mu_{\zeta}^{2} v_{2}=0,
\end{aligned}
$$

From Eq. 6 we find three sets of solutions 


$$
\begin{aligned}
& v_{1}=v_{2}=0 \\
& \mu_{S M}=-a_{4} v_{S M}^{2} \\
& v_{2}=-v_{1}, \quad \mu_{\zeta}^{2}=\frac{1}{2}\left(-b_{1} v_{S M}^{2}-4 v_{1}^{2}\left(c_{1}+c_{2}\right)+\mu_{12}^{2}\right), \\
& \mu_{S M}=-a_{4} v_{S M}^{2}-b_{1} v_{1}^{2} \\
& v_{2}=v_{1}, \quad \mu_{\zeta}^{2}=\frac{1}{2}\left(-b_{1} v_{S M}^{2}-4 v_{1}^{2}\left(c_{1}+c_{2}\right)-\mu_{12}^{2}\right), \\
& \mu_{S M}=-a_{4} v_{S M}^{2}-b_{1} v_{1}^{2}
\end{aligned}
$$

These solutions will be used in the analyses presented throughout this work.

\section{Mass matrices and eigenstates}

The potential in Eq. 4 gives us four mass matrices: one for the charged scalars, one for the CP-odd neutral scalars and

$$
\left(\begin{array}{c}
S^{+} \\
D_{1}^{+} \\
D_{2}^{+}
\end{array}\right)=R_{C}\left(\begin{array}{l}
G^{+} \\
H_{1}^{+} \\
H_{2}^{+}
\end{array}\right)=\left(\begin{array}{ccc}
1 & 0 & 0 \\
0 & \pm \frac{1}{\sqrt{2}} & \mp \frac{1}{\sqrt{2}} \\
0 & \frac{1}{\sqrt{2}} & \frac{1}{\sqrt{2}}
\end{array}\right)\left(\begin{array}{l}
G^{+} \\
H_{1}^{+} \\
H_{2}^{+}
\end{array}\right) .
$$

And their masses are:

$$
\begin{aligned}
& m_{G^{+}}^{2}=0, \quad m_{H_{1}^{+}}^{2}=\frac{a_{5} v_{S M}^{2}}{2}+v_{1}^{2}\left(b_{2}-2 b_{3}\right)+\mu_{d}^{2}, \\
& m_{H_{2}^{+}}^{2}=\frac{a_{5} v_{S M}^{2}}{2}+v_{1}^{2}\left(b_{2}+2 b_{3}\right)+\mu_{d}^{2} .
\end{aligned}
$$

\subsection{CP-odd scalars}

From Eq. 5, in the basis $\left(\operatorname{Im}\left[S^{0}\right], \chi_{1}, \chi_{2}\right) M_{O}\left(\operatorname{Im}\left[S^{0}\right], \chi_{1}\right.$, $\left.\chi_{2}\right)^{T}$, we find the mass matrix $M_{O}$ for the CP-odd scalars to be

$$
M_{O}=\left(\begin{array}{lcc}
0 & 0 & 0 \\
0 b_{2} v_{1}^{2}+\frac{1}{2}\left(a_{5}-2 a_{6}+a_{7}\right) v_{S M}^{2}+\mu_{d}^{2} & \mp 2 b_{3} v_{1}^{2} \\
0 & \mp 2 b_{3} v_{1}^{2} & b_{2} v_{1}^{2}+\frac{1}{2}\left(a_{5}-2 a_{6}+a_{7}\right) v_{S M}^{2}+\mu_{d}^{2}
\end{array}\right) \text {. }
$$

two for the CP-even neutral scalars. In the sections below we show these matrices and their corresponding eigenvalues and eigenvectors. Also, when we have \pm or $\mp$, the upper sign corresponds to the vacuum stability criteria from Eq. 8 and the lower sign to Eq. 9.

\subsection{Charged scalars}

From Eq. 5 , in the basis $\left(S^{+}, D_{1}^{+}, D_{2}^{+}\right) M_{C}\left(S^{-}, D_{1}^{-}, D_{2}^{-}\right)^{T}$, we find the mass matrix $M_{C}$ for the charged scalars to be

$$
M_{C}=\left(\begin{array}{lcc}
0 & 0 & 0 \\
0 b_{2} v_{1}^{2}+\frac{a_{5} v_{S M}^{2}}{2}+\mu_{d}^{2} & \mp 2 b_{3} v_{1}^{2} \\
0 & \mp 2 b_{3} v_{1}^{2} & b_{2} v_{1}^{2}+\frac{a_{5} v_{S M}^{2}}{2}+\mu_{d}^{2}
\end{array}\right) \text {. }
$$

The above matrix can be diagonalized as $R_{C}^{T} M_{C} R_{C}$, where $R_{C}$ is the orthogonal rotation matrix. For the matrix in Eq. 10
The diagonalization for this mass matrix is given by

$$
\left(\begin{array}{c}
\operatorname{Im}\left[S^{0}\right] \\
\chi_{1} \\
\chi_{2}
\end{array}\right)=R_{O}\left(\begin{array}{c}
G_{A}^{0} \\
A_{1}^{0} \\
A_{2}^{0}
\end{array}\right)=\left(\begin{array}{ccc}
1 & 0 & 0 \\
0 & \pm \frac{1}{\sqrt{2}} & \mp \frac{1}{\sqrt{2}} \\
0 & \frac{1}{\sqrt{2}} & \frac{1}{\sqrt{2}}
\end{array}\right)\left(\begin{array}{c}
G_{A}^{0} \\
A_{1}^{0} \\
A_{2}^{0}
\end{array}\right) .
$$

With masses:

$$
\begin{gathered}
m_{G_{A}^{0}}^{2}=0, \quad m_{A_{1}^{0}}^{2}=\frac{1}{2} v_{S M}^{2}\left(a_{5}-2 a_{6}+a_{7}\right)+v_{1}^{2}\left(b_{2}-2 b_{3}\right)+\mu_{d}^{2}, \\
m_{A_{2}^{0}}^{2}=\frac{1}{2} v_{S M}^{2}\left(a_{5}-2 a_{6}+a_{7}\right)+v_{1}^{2}\left(b_{2}+2 b_{3}\right)+\mu_{d}^{2} .
\end{gathered}
$$

\subsection{CP-even scalars, $2 \times 2$ matrix}

Equation 5 gives us for the CP-even sector two matrices, one $2 \times 2$ and one $3 \times 3$. The $2 \times 2$ matrix, in the basis $\left(\eta_{1}, \eta_{2}\right) M_{E 1}\left(\eta_{1}, \eta_{2}\right)^{T}$, is

$$
M_{E 1}=\left(\begin{array}{cc}
b_{2} v_{1}^{2}+\frac{1}{2}\left(a_{5}+2 a_{6}+a_{7}\right) v_{S M}^{2}+\mu_{d}^{2} & \mp 2 b_{3} v_{1}^{2} \\
\mp 2 b_{3} v_{1}^{2} & b_{2} v_{1}^{2}+\frac{1}{2}\left(a_{5}+2 a_{6}+a_{7}\right) v_{S M}^{2}+\mu_{d}^{2}
\end{array}\right) \text {. }
$$

we find that the symmetry and mass eigenstates are related as
The symmetry and mass eigenstates are related as 


$$
\left(\begin{array}{l}
\eta_{1} \\
\eta_{2}
\end{array}\right)=R_{E 1}\left(\begin{array}{l}
h_{1}^{0} \\
h_{2}^{0}
\end{array}\right)=\left(\begin{array}{cc} 
\pm \frac{1}{\sqrt{2}} \mp \frac{1}{\sqrt{2}} \\
\frac{1}{\sqrt{2}} & \frac{1}{\sqrt{2}}
\end{array}\right)\left(\begin{array}{l}
h_{1}^{0} \\
h_{2}^{0}
\end{array}\right) .
$$

The masses are:

$$
\begin{aligned}
& m_{h_{1}^{0}}^{2}=\frac{1}{2} v_{S M}^{2}\left(a_{5}+2 a_{6}+a_{7}\right)+v_{1}^{2}\left(b_{2}-2 b_{3}\right)+\mu_{d}^{2}, \\
& m_{h_{2}^{0}}^{2}=\frac{1}{2} v_{S M}^{2}\left(a_{5}+2 a_{6}+a_{7}\right)+v_{1}^{2}\left(b_{2}+2 b_{3}\right)+\mu_{d}^{2} .
\end{aligned}
$$

$$
\begin{aligned}
& \left(\begin{array}{c}
H \\
H_{1}^{0} \\
H_{2}^{0}
\end{array}\right)=R_{E 2}^{T}\left(\begin{array}{c}
\operatorname{Re}\left[S^{0}\right] \\
\xi_{1} \\
\xi_{2}
\end{array}\right) \\
& =\left(\begin{array}{ccc}
\cos \theta_{2} & -\cos \theta_{3} \sin \theta_{2} & \sin \theta_{2} \sin \theta_{3} \\
\cos \theta_{1} \sin \theta_{2} & \cos \theta_{1} \cos \theta_{2} \cos \theta_{3}-\sin \theta_{1} \sin \theta_{3} & -\cos \theta_{3} \sin \theta_{1}-\cos \theta_{1} \cos \theta_{2} \sin \theta_{3} \\
\sin \theta_{1} \sin \theta_{2} & \cos \theta_{2} \cos \theta_{3} \sin \theta_{1}+\cos \theta_{1} \sin \theta_{3} & \cos \theta_{1} \cos \theta_{3}-\cos \theta_{2} \sin \theta_{1} \sin \theta_{3}
\end{array}\right)\left(\begin{array}{c}
R e\left[S^{0}\right] \\
\xi_{1} \\
\xi_{2}
\end{array}\right) .
\end{aligned}
$$

(right-handed charged lepton singlets), $G^{\prime} s$ are the Yukawa couplings, and $N_{s, d}$ are the right-handed neutrinos.

From Eq. 20, $\operatorname{Re}\left[S^{0}\right]$ is the only one that gives mass to the known fermions, therefore we will identify it as the SM Higgs. The matrix in Eq. 19 can be diagonalized by an orthogonal $3 \times 3$ matrix, such that $R_{E 2}^{T} M_{E 2} R_{E 2}=$ $\operatorname{diag}\left(m_{H}^{2}, m_{H_{1}^{0}}^{2}, m_{H_{2}^{0}}^{2}\right)$, where $H$ is the SM Higgs boson and $H_{1,2}^{0}$ are the other CP-even scalars from the $3 \times 3$ CP-even mass matrix. This implies

\section{The CP-even 3 x 3 matrix and the Higgs boson}

Again from Eq. 5, we obtain the $3 \times 3$ matrix for the CP-even scalars. Considering the basis $\left(\operatorname{Re}\left[S^{0}\right], \xi_{1}, \xi_{2}\right) M_{E 2}\left(\operatorname{Re}\left[S^{0}\right]\right.$, $\left.\xi_{1}, \xi_{2}\right)$ we find

$M_{E 2}=\left(\begin{array}{ccc}2 a_{4} v_{S M}^{2} & b_{1} v_{1} v_{S M} & \mp b_{1} v_{1} v_{S M} \\ b_{1} v_{1} v_{S M} & \frac{1}{2}\left(4\left(c_{1}+c_{2}\right) v_{1}^{2} \pm \mu_{12}^{2}\right) & \frac{1}{2}\left(\mu_{12}^{2} \mp 4\left(c_{1}+c_{2}\right) v_{1}^{2}\right) \\ \mp b_{1} v_{1} v_{S M} & \frac{1}{2}\left(\mu_{12}^{2} \mp 4\left(c_{1}+c_{2}\right) v_{1}^{2}\right) & \frac{1}{2}\left(4\left(c_{1}+c_{2}\right) v_{1}^{2} \pm \mu_{12}^{2}\right)\end{array}\right)$,

where, when we have \pm or $\mp$, the upper sign corresponds to the vacuum stability criteria from Eq. 8 and the lower sign to Eq. 9. To find the mass eigenstates of this sector, we will follow Ref. [36], where we impose that the Yukawa couplings of the Higgs boson are the same in the SM and in the $S_{3} \otimes \mathbb{Z}_{2}$ model.

In the $S_{3} \otimes \mathbb{Z}_{2}$ model, the Yukawa sector for the leptons is given by

$$
\begin{aligned}
-\mathcal{L}_{\text {Yukawa }}^{\text {leptons }}= & G_{i j}^{l} \bar{L}_{i} l_{j R} S+G_{i j}^{v} L_{i} v_{j L} S+G_{i s}^{N} L_{i} \in N_{s R} S \\
& +\frac{y_{d}}{2}\left[\left[\overline{N_{d R}^{c}} N_{d R}\right]_{2} \zeta_{d}\right]_{1}+\frac{y_{s d}}{2}\left[\overline{N_{s R}^{c}}\left[N_{d R} \zeta_{d}\right]_{2}\right]_{1} \\
& +\frac{F_{i s}^{v}}{\Lambda} \bar{L}^{c}{ }_{i} N_{S}\left[D_{d} \zeta_{d}^{*}\right]_{1}+\text { H.c. },
\end{aligned}
$$

where $i, j=e, \mu, \tau, d=1,2$ (we omit summation symbols), $L_{i}\left(l_{i R}\right)$ denote the usual left-handed lepton doublets
Since $\operatorname{Re}\left[S^{0}\right]$ is the scalar we identify as the SM Higgs, we need that $\left(R_{E 2}^{T}\right)_{11}=1$ and all the other elements from the first row to be zero. To do so, we need $\theta_{2}=0$, which gives us $\cos \theta_{2}=1$ and $\sin \theta_{2}=0$, leaving $R_{E 2}$ as

$$
\begin{aligned}
R_{E 2} & =\left(\begin{array}{ccc}
1 & 0 & 0 \\
0 & \cos \theta_{1} \cos \theta_{3}-\sin \theta_{1} \sin \theta_{3} & \cos \theta_{3} \sin \theta_{1}+\cos \theta_{1} \sin \theta_{3} \\
0 & -\cos \theta_{3} \sin \theta_{1}-\cos \theta_{1} \sin \theta_{3} & \cos \theta_{1} \cos \theta_{3}-\sin \theta_{1} \sin \theta_{3}
\end{array}\right) \\
& =\left(\begin{array}{ccc}
1 & 0 & 0 \\
0 & \cos \theta & \sin \theta \\
0 & -\sin \theta & \cos \theta
\end{array}\right),
\end{aligned}
$$

where $\theta=\theta_{1}+\theta_{3}$. The matrix $R_{E 2}$ from Eq. 22 does not automatically diagonalize $M_{E 2}$, i.e., it does not lead to $R_{E 2}^{T} M_{E 2} R_{E 2}=\operatorname{diag}\left(m_{H}^{2}, m_{H_{1}^{0}}^{2}, m_{H_{2}^{0}}^{2}\right)$. To have that we need to impose conditions on the parameters that make up $M_{E 2}$ and $R_{E 2}$, so that we fulfill the equation $R_{E 2}^{T} M_{E 2} R_{E 2}=$ $\operatorname{diag}\left(m_{H}^{2}, m_{H_{1}^{0}}^{2}, m_{H_{2}^{0}}^{2}\right)$. The possible solutions depend on which solution for the vacuum stability conditions (Eqs. 7-9) we choose.

1. $v_{1}=v_{2}=0$ :

This solution trivializes our mass matrix $M_{E 2}$, making it diagonal from the very beginning, leaving one scalar with mass $m_{R e\left[S^{0}\right]}^{2}=a_{4} v_{S M}^{2}$ and the other two scalars with mass $m_{\xi_{1}, \xi_{2}}^{2}=-\mu_{12}^{2} / 4$. Therefore, there is no need for the diagonalization method presented in this section.

2. $v_{2}=-v_{1}$ and $\mu_{\zeta}^{2}=\frac{1}{2}\left(-b_{1} v_{S M}^{2}-4 v_{1}^{2}\left(c_{1}+c_{2}\right)+\mu_{12}^{2}\right)$ : 
In this case we find 10 possible solutions for the parameters $a_{4}, b_{1}, \mu_{12}^{2}$ and the sines and cosines of $\theta$. Amongst all solutions, we either have all masses equal, $m^{2}=$ $4 v_{1}^{2}\left(c_{1}+c_{2}\right)$; or two equal masses, $m_{1}^{2}=2 a_{4} v_{S M}^{2}$, and a third different mass $m_{2}^{2}=4 v_{1}^{2}\left(c_{1}+c_{2}\right)$. In both cases, $b_{1}=0$. When all masses are equal, $a_{4}=\frac{2 v_{1}^{2}\left(c_{1}+c_{2}\right)}{v_{S M}^{2}}$ and $\mu_{12}^{2}=4 v_{1}^{2}\left(c_{1}+c_{2}\right)$; when we have different masses, $\mu_{12}^{2}=2 a_{4} v_{S M}^{2}$. As for the sines and cosines, they can either be functions of each other or have values $\pm 1 / \sqrt{2}$.

3. $v_{2}=v_{1}, \quad \mu_{\zeta}^{2}=\frac{1}{2}\left(-b_{1} v_{S M}^{2}-4 v_{1}^{2}\left(c_{1}+c_{2}\right)-\mu_{12}^{2}\right)$ :

Once again we have 10 possible solutions for the parameters $a_{4}, b_{1}, \mu_{12}^{2}$ and the sines and cosines of $\theta$. Amongst all solutions, we either have all masses equal, $m^{2}=$ $4 v_{1}^{2}\left(c_{1}+c_{2}\right)$; or two equal masses, $m_{1}^{2}=2 a_{4} v_{S M}^{2}$, and a third different mass $m_{2}^{2}=4 v_{1}^{2}\left(c_{1}+c_{2}\right)$. In both cases, $b_{1}=0$. When all masses are equal, $a_{4}=\frac{2 v_{1}^{2}\left(c_{1}+c_{2}\right)}{v_{S M}^{2}}$ and $\mu_{12}^{2}=-4 v_{1}^{2}\left(c_{1}+c_{2}\right)$; when we have different masses, $\mu_{12}^{2}=-2 a_{4} v_{S M}^{2}$. As for the sines and cosines, they can either be functions of each other or have values $\pm 1 / \sqrt{2}$.

The complete set of solutions are shown in Appendix A.

\section{The $e^{-} e^{+} \rightarrow H^{+} H^{-}$process at CLIC}

The lowest order contributions to the $e^{-} e^{+} \rightarrow H^{+} H^{-}$process in our model are given by the diagrams in Fig. 1. For the scalar mass eigenstates we considered solution 10 from the Appendix A 2. The model has two charged scalars, $H_{1}^{ \pm}$ and $H_{2}^{ \pm}$. In here we assumed that $H_{1}^{ \pm}$is the lightest one, and that is the one considered in our cross sections. Taking these assumptions into account, the relevant vertices for our calculations are:

$$
\begin{aligned}
& H^{+} H^{-} Z \rightarrow-\sqrt{\pi \alpha} \frac{c_{W}^{2}-s_{W}^{2}}{c_{W} S_{W}}, \\
& H^{+} H^{-} \gamma \rightarrow \sqrt{4 \pi \alpha}, \\
& e^{ \pm} N H^{ \pm} \rightarrow \sqrt{2} F_{1 S}^{v} \frac{v_{1}}{\Lambda}=\sqrt{2} X, \\
& H H^{+} H^{-} \rightarrow a_{5} v_{S M}, \\
& e^{+} e^{-} H \rightarrow \frac{m_{e}}{v_{S M}} ;
\end{aligned}
$$

where $\alpha$ is the fine structure constant, $v_{S M}$ is the SM vacuum expectation value for the Higgs field, $m_{e}$ is the electron mass, and $c_{W}$ and $s_{W}$ are the cosine and sine of the electroweak angle, respectively. $F_{1 S}^{v}$ and $\Lambda$ are parameters of the Yukawa sector of our model (Eq. 2), and $v_{1}$ and $a_{5}$ are parameters of the scalar sector of our model.

To calculate the cross sections we considered the three energy stages that CLIC will run at: $380 \mathrm{GeV}, 1.5 \mathrm{TeV}$ and 3

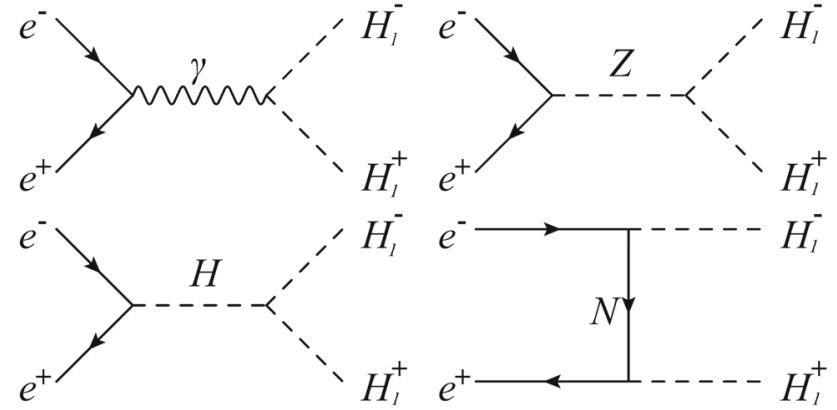

Fig. 1 Lowest order contributions to the $e^{-} e^{+} \rightarrow H^{+} H^{-}$process

Table 1 Baseline CLIC energy stages and integrated luminosities for each stage [33]

\begin{tabular}{lll}
\hline Stage & $\sqrt{s}(\mathrm{TeV})$ & $\mathcal{L}_{\text {int }}\left(a b^{-1}\right)$ \\
\hline 1 & 0.38 & 1.0 \\
2 & 1.5 & 2.5 \\
3 & 3 & 5.0 \\
\hline
\end{tabular}

TeV (see Table 1). Throughout this section we will consider $X \equiv v_{1} F_{1 s}^{v} / \Lambda=0.1,0.01,10^{-6}$ and $10^{-7}$ (these values for $X$ allow perturbative calculations). Also, we will consider $a_{5}=1$ for the coupling constant in the $\mathrm{HH}_{1}^{+} H_{1}^{-}$vertex, the highest possible value that allows perturbative calculations.

In Fig. 2 are presented the $e^{+} e^{-} \rightarrow H_{1}^{+} H_{1}^{-}$cross section predictions for $\sqrt{s}=380 \mathrm{GeV}$. The top left graph shows that the contribution of the sterile neutrino to the cross section is constant in the range $m_{N}=[1,1000] \mathrm{eV}$, therefore we set $m_{N}=1 \mathrm{eV}$ in the other plots, which is compatible with the current experimental data [37]. In the top right graph the partial contributions show the hierarchy of the contributors: the photon is the main responsible, it is followed by the sterile neutrino with $m_{N}=1 \mathrm{eV}$ and $X=0.1$, the third place is due to the $Z$ gauge boson, then the signal of the neutrino with $X=0.01$ is suppressed, followed the Higgs boson, finally, the neutrino signals with $X=10^{-6}$ and $X=10^{-7}$ with the lowest contribtions, several orders of magnitude below the photon one. At the bottom we show the total cross section, taking into account all diagrams from Fig. 1. The graphs for $X=0.01, X=10^{-6}$ and $X=10^{-7}$ are all superposed, given the negligible contributions for $X \leq 0.1$. One can notice that for $0 \mathrm{GeV}<m_{H_{1}^{+}}<170 \mathrm{GeV}$ the total cross section stays roughly in the interval $\sigma=[0.1 \mathrm{pb}, 0.005 \mathrm{pb}]$.

In Fig. 3 are presented the $e^{+} e^{-} \rightarrow H_{1}^{+} H_{1}^{-}$cross section predictions for $\sqrt{s}=1.5 \mathrm{TeV}$. The top left graph shows that the contribution of the sterile neutrino to the cross section is once again constant in the range $m_{N}=[1,1000] \mathrm{eV}$, therefore we set $m_{N}=1 \mathrm{eV}$ in the other plots. In the top right graph the partial contributions follow the same hierarchy from Fig. 2, this time however, the cross sections are about one order of magnitude smaller than before. At the bottom we 


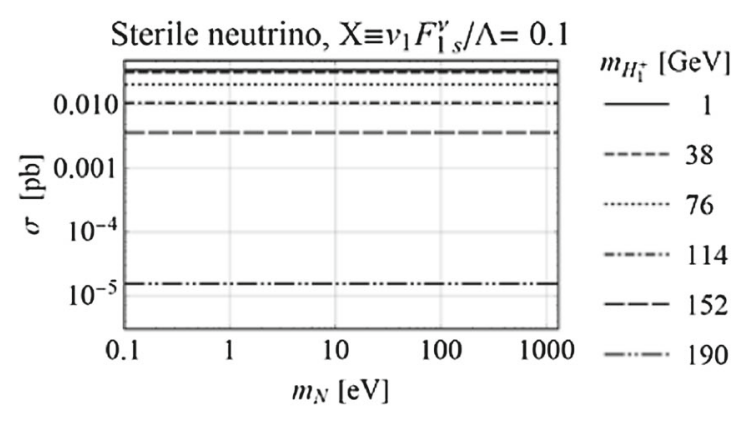

(a)

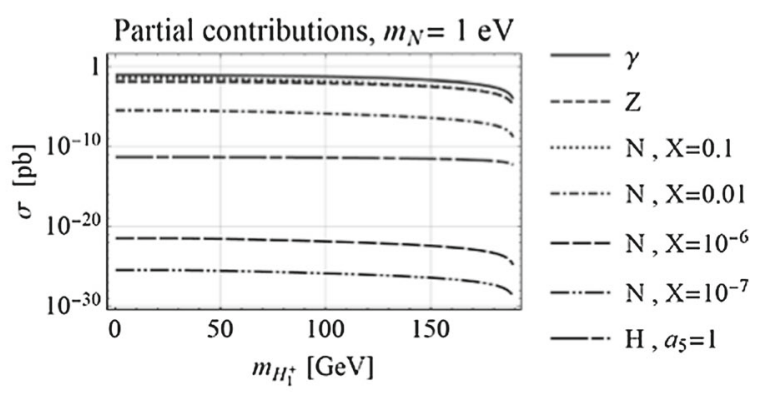

(b)

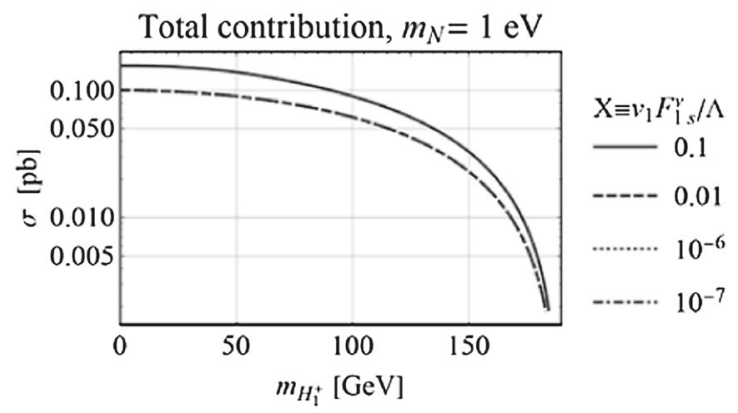

(c)

Fig. 2 Predictions for the $e^{+} e^{-} \rightarrow H_{1}^{+} H_{1}^{-}$cross section with $\sqrt{s}=$ $380 \mathrm{GeV}$. a The sterile neutrino contribution for the cross-section as a function of its mass, considering several mass values for the charged scalar. $\mathbf{b}$ The partial contribution to the cross section as a function of the charged scalar mass. $\mathbf{c}$ The total cross section as a function of the charged scalar mass

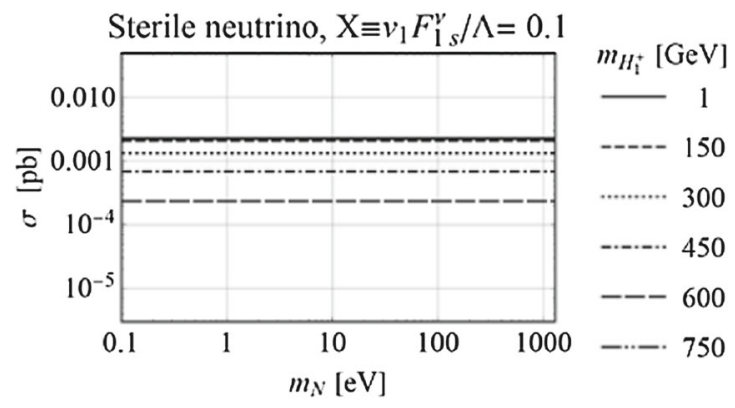

(a)

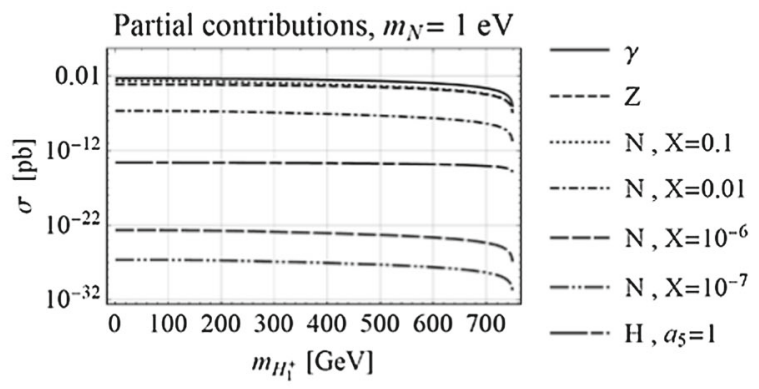

(b)

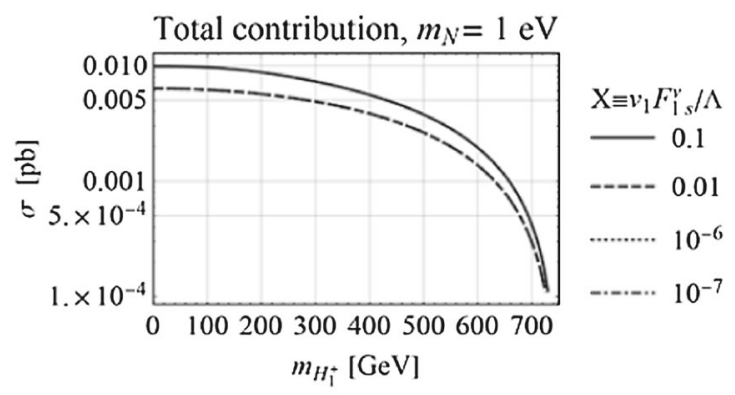

(c)

Fig. 3 Predictions for the $e^{+} e^{-} \rightarrow H_{1}^{+} H_{1}^{-}$cross section with $\sqrt{s}=$ 1.5 TeV. a The sterile neutrino contribution for the cross-section as a function of its mass, considering several mass values for the charged scalar. b The partial contribution to the cross section as a function of the charged scalar mass. c The total cross section as a function of the charged scalar mass 


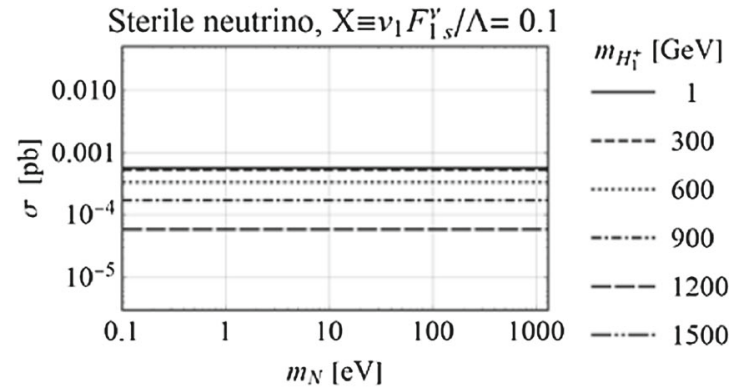

(a)

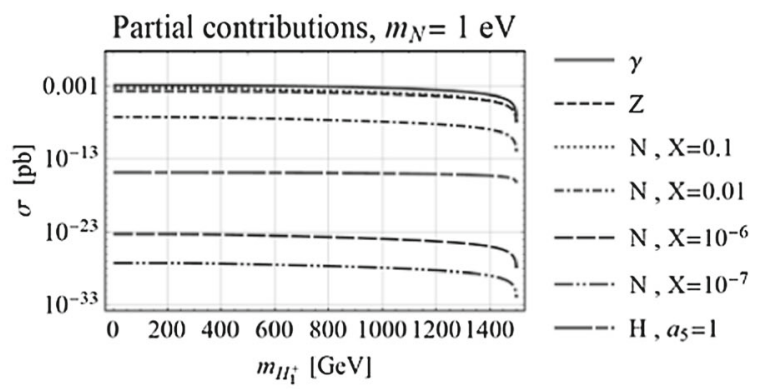

(b)

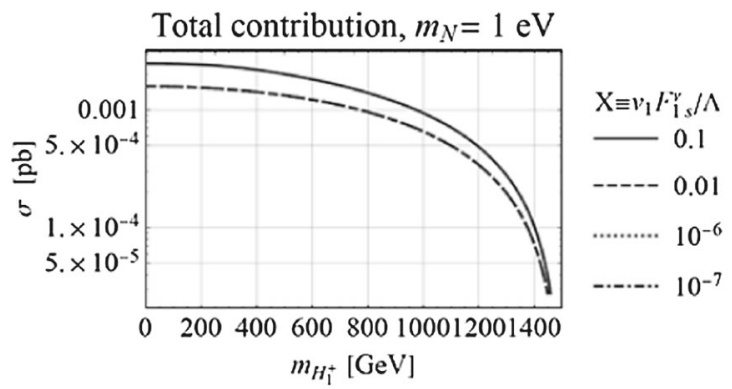

(c)

Fig. 4 Predictions for the $e^{+} e^{-} \rightarrow H_{1}^{+} H_{1}^{-}$cross section with $\sqrt{s}=$ $3 \mathrm{TeV}$. a The sterile neutrino contribution for the cross-section as a function of its mass, considering several mass values for the charged scalar. b The partial contribution to the cross section as a function of the charged scalar mass. c The total cross section as a function of the charged scalar mass show the total cross section, taking into account all diagrams from Fig. 1. One can notice that for $0 \mathrm{GeV}<m_{H_{1}}<700$ $\mathrm{GeV}$ the total cross section stays in the interval $\sigma=[0.01 \mathrm{pb}$, $0.0001 \mathrm{pb}$ ], about one order of magnitude smaller than in the $\sqrt{s}=380 \mathrm{GeV}$ case and the individual contributions to the total cross section for $X=0.01, X=10^{-6}$ and $X=10^{-7}$ are all superposed.

In Fig. 4 are presented the $e^{+} e^{-} \rightarrow H_{1}^{+} H_{1}^{-}$cross section predictions for $\sqrt{s}=3 \mathrm{TeV}$. Just like in the previous cases, the top left graph shows that the contribution of the sterile neutrino to the cross section is constant in the range $m_{N}=$ $[1,1000] \mathrm{eV}$. In the top right graph the partial contributions follow the same hierarchy from Figs. 2 and 3, where the cross sections are about one order of magnitude smaller than the $\sqrt{s}=1.5 \mathrm{TeV}$ case. At the bottom we show the total cross section, taking into account all diagrams from Fig. 1, where. One can notice that for $0 \mathrm{GeV}<m_{H_{1}}<1400 \mathrm{GeV}$ the total cross section stays roughly in the interval $\sigma=[0.01 \mathrm{pb}$, $5 \times 10^{-5} \mathrm{pb}$, about one order of magnitude below than the $\sqrt{s}=1.5 \mathrm{TeV}$ case.

Overall, we can expect a cross section for the $e^{+} e^{-} \rightarrow$ $H_{1}^{+} H_{1}^{-}$process roughly in between $0.1 \mathrm{pb}$ and $5 \times 10^{-5} \mathrm{pb}$ (depending on $\sqrt{s}$ and on $m_{H_{1}}$ ). This is true even if we set $X=a_{5}=0$, decoupling the sterile neutrino and the Higgs boson from the charged scalars, given that the contributions from the photon and $Z$ boson are predominant in this case.
Table 2 Transformation properties of the fermion and scalar fields under $S_{3}$ and $\mathbb{Z}_{2}$ symmetries. Quarks and charged leptons are singlets of $S_{3}$ and even under $\mathbb{Z}_{2}$

\begin{tabular}{llllllll}
\hline Symmetry & $L_{i}$ & $l_{j R}$ & $N_{s}$ & $N_{d}$ & $S$ & $D$ & $\zeta_{d}$ \\
\hline$S_{3}$ & 1 & 1 & 1 & 2 & 1 & 2 & 2 \\
$\mathbb{Z}_{2}$ & 1 & -1 & 1 & 1 & -1 & 1 & 1 \\
\hline
\end{tabular}

Other Standard Model extensions with charged scalars are likely to give similar results if $\mathrm{H}^{+} \mathrm{H}^{-} \mathrm{Z}$ and $\mathrm{H}^{+} \mathrm{H}^{-} \gamma$ vertices are present. Also, had we considered other solutions for the scalar mass eigenstates, it would only change the $e^{ \pm} \mathrm{NH}^{ \pm}$and $\mathrm{HH}^{+} \mathrm{H}^{-}$vertices, introducing mixing angles that are likely to reduce these vertices. Therefore, changes in these vertices would give negligible differences in our total cross section prediction given that the main contribution comes from the photon, which has its vertex unchanged from choosing other solutions for the scalar mass eigenstates.

Considering the integrated luminosities shown in Table 1, for $\sqrt{s}=380 \mathrm{GeV}$, where the cross section ranges in between $0.1 \mathrm{pb}$ and $0.005 \mathrm{pb}$, the first stage of CLIC should give us a number of events in between $10^{5}$ and $5 \times 10^{3}$. Meanwhile, for stage 2, with our cross section predictions ranging from $0.01 \mathrm{pb}$ up to $0.0001 \mathrm{pb}$, the number of events should be between $2.5 \times 10^{4}$ and 250 . Finally, for $\sqrt{s}=3 \mathrm{TeV}$, where 
Table 3 Cross-sections for the $e \bar{e} \rightarrow e \bar{e} N \bar{N}$ process with $\sqrt{s}=$ $380 \mathrm{GeV}$. The cross-section values are in picobarns

Table 4 Cross-sections for the $e \bar{e} \rightarrow e \bar{e} N \bar{N}$ process with $\sqrt{s}=$ $1500 \mathrm{GeV}$. The cross-section values are in picobarns

Table 5 Cross-sections for the $e \bar{e} \rightarrow e \bar{e} N \bar{N}$ process with $\sqrt{s}=$ $3000 \mathrm{GeV}$. The cross-section values are in picobarns

\begin{tabular}{lllll}
\hline$\sqrt{s}=380 \mathrm{GeV}$ & $m_{H_{1}^{+}}=2 \mathrm{GeV}$ & $m_{H_{1}^{+}}=10 \mathrm{GeV}$ & $m_{H_{1}^{+}}=100 \mathrm{GeV}$ & $m_{H_{1}^{+}}=1000 \mathrm{GeV}$ \\
\hline$m_{N}=10^{-9} \mathrm{GeV}$ & $4.81 \times 10^{-2}$ & $7.57 \times 10^{-2}$ & $1.03 \times 10^{-3}$ & $1.16 \times 10^{-8}$ \\
\hline
\end{tabular}

\begin{tabular}{lllll}
\hline$\sqrt{s}=1500 \mathrm{GeV}$ & $m_{H_{1}^{+}}=2 \mathrm{GeV}$ & $m_{H_{1}^{+}}=10 \mathrm{GeV}$ & $m_{H_{1}^{+}}=100 \mathrm{GeV}$ & $m_{H_{1}^{+}}=1000 \mathrm{GeV}$ \\
\hline$m_{N}=10^{-9} \mathrm{GeV}$ & $2.17 \times 10^{-3}$ & $6.31 \times 10^{-3}$ & $3.10 \times 10^{-4}$ & $1.25 \times 10^{-6}$ \\
\hline
\end{tabular}

\begin{tabular}{lllll}
\hline$\sqrt{s}=3000 \mathrm{GeV}$ & $m_{H_{1}^{+}}=2 \mathrm{GeV}$ & $m_{H_{1}^{+}}=10 \mathrm{GeV}$ & $m_{H_{1}^{+}}=100 \mathrm{GeV}$ & $m_{H_{1}^{+}}=1000 \mathrm{GeV}$ \\
\hline$m_{N}=10^{-9} \mathrm{GeV}$ & $4.90 \times 10^{-4}$ & $3.06 \times 10^{-3}$ & $3.43 \times 10^{-4}$ & $4.27 \times 10^{-5}$ \\
$m_{N}=1 \mathrm{GeV}$ & $7.30 \times 10^{-3}$ & $2.34 \times 10^{-2}$ & $1.55 \times 10^{-4}$ & $3.65 \times 10^{-5}$ \\
\hline
\end{tabular}

we predict $\sigma=\left[0.01 \mathrm{pb}, 5 \times 10^{-5} \mathrm{pb}\right]$, the number of events should be between $5 \times 10^{4}$ and 50 .

\subsection{The $H_{1}^{ \pm}$decay}

At first, one would expect to detect these exotic charged scalars through their decay products, which would leave their signals in the detector. The main decay for $H_{1}^{+}$is $H_{1}^{+} \rightarrow \bar{e} N$, which leads to $e \bar{e} \rightarrow e \bar{e} N \bar{N}$ in an electron-positron collider, where the main background for this process in the SM comes from $e \bar{e} \rightarrow e \bar{e} \nu_{e} \bar{\nu}_{e}$.

At a glance, one would expect the $H_{1}^{+} \rightarrow \bar{e} N H$ and $H_{1}^{+} \rightarrow W^{+} H$ decays as well (where $H$ is the SM higgs boson), however, there are no vertices involving $H_{1}^{ \pm}$and $H$, which forbids these processes. This can also be seen from Table 2, the exotic particles are charged under the $S_{3}$ and $Z_{2}$ symmetries, while the SM particles aren't, therefore forbidding these decays.

In the $\mathrm{SM} \sigma\left(e \bar{e} \rightarrow e \bar{e} \nu_{e} \bar{\nu}_{e}\right)_{\mathrm{SM}}=4.41 \times 10^{-1}, 5.00 \times$ $10^{-1}, 1 \mathrm{pb}$ for the center of mass energies $\sqrt{s}=380,1500$, $3000 \mathrm{GeV}$, while in our $S_{3}$ model $\sigma(e \bar{e} \rightarrow e \bar{e} N \bar{N})_{S 3}$ crosssections, assuming $X=v_{1} F_{1, s}^{v} / \Lambda=0.1$, can be seen in Tables 3, 4 and 5. From the tables one can see that as $m_{H_{1}^{+}}$ increases, so does the cross-section, while the opposite is true with the increase in $\sqrt{s}$. This cross-section behavior with the increase in $\sqrt{s}$ differs in the SM, where it increases with the increase in energy. Therefore, for lower values of $\sqrt{s}$ and $m_{H_{1}^{+}}$one may see a noticeable increase in $e \bar{e}+$ missing energy events. In other situations, we expect this signal to be dominated by the SM. Also, we considered the highest possible value for $X$ that allows perturbative calculations, lower values would imply even smaller cross-sections.

Given the massive background signal of the $e \bar{e} \rightarrow e \bar{e} N \bar{N}$ channel, we are left with the possibility of a direct detection, where the charged scalars hit the inner tracker of the detector. The innermost tracker layer is at $165 \mathrm{~mm}$ from the center [38], therefore, that is the minimal travel distance necessary for the charged scalar to be directly detected.

In Figs. 5 and 6 we show the total travel distance of the $\mathrm{H}_{1}^{+}$scalar in the detector, taking into account different mass values for the exotic neutrino $N$, the coupling constant $X$, and relativistic time dilation effects on the lifetime of $H_{1}^{+}$. In this case we assumed that the couplings in the $H_{1}^{+} \bar{N} e, H_{1}^{+} \bar{N} \mu$ and $H_{1}^{+} \bar{N} \tau$ vertices are all the same, giving us a single value for $X$, instead of a different value for each coupling. We considered $X=10^{-6}$ and $X=10^{-7}$ to increase the lifetime of $\mathrm{H}_{1}^{+}$, where larger values of $X$ would lead to short lifetimes, making it impossible for the scalar to reach the inner tracker of the detector.

One can see that when $m_{H_{1}^{+}} \approx m_{N}$, the exotic charged scalar is able to reach the detector, making it possible for a direct detection. Also, the smaller $X$ is, the more likely the detection, given that $m_{H_{1}^{+}}$and $m_{N}$ don't have to be as close. Comparing the values for $X$ in Figs. 5 and 6 with the ones used for the cross-section calculations (Figs. 2, 3, 4), the ones used for the travel distance are orders of magnitude smaller, which in consequence, reduces the total crosssection. However, given that the diagrams mediated by $N$ are not the main contributors to the total cross-section, specially for $X=10^{-6}$ and $X=10^{-7}$, the overall reduction in events is insignificant. In the end, there are a variety of factors that determine whether or not the scalar can be directly detected: the mass values of $H_{1}^{+}$and $N$, the coupling constant $X$ and the energy of the collisions.

\section{Conclusions}

We have calculated the cross-section at tree-level for the production of charged scalars in eletron-positron collisions in the $S_{3} \otimes \mathbb{Z}_{2}$ model. For our numerical results we considered the compact linear collider energies and luminosities, so we could have a realistic view of possible experimental 


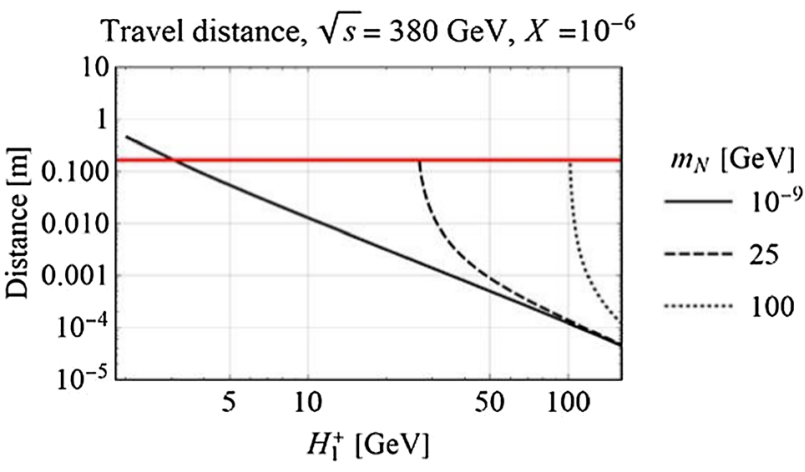

(a)

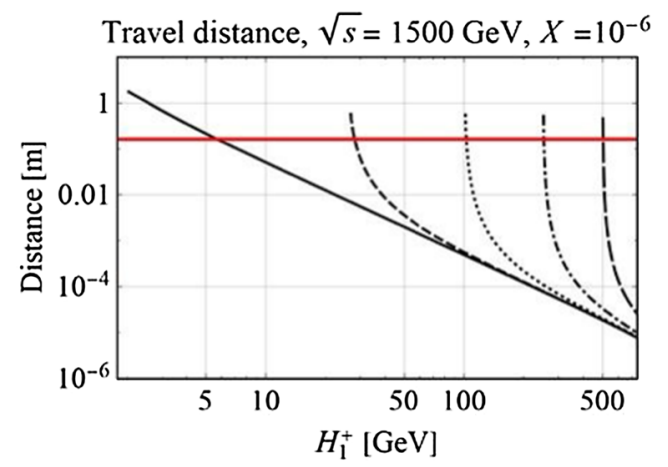

(b)

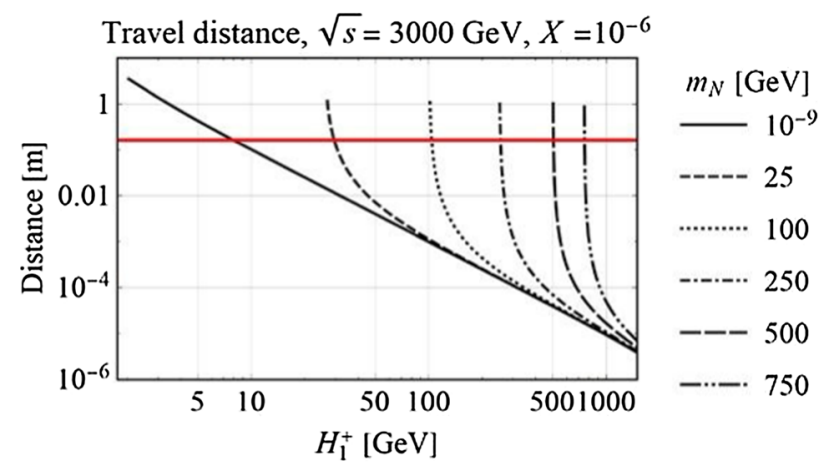

(c)

Fig. $5 H_{1}^{+}$travel distance. The graphs show the total travel distance of $H_{1}^{+}$for different values of $m_{N}$ and energy stages of CLIC (a $\sqrt{s}: 380$ $\mathrm{GeV}, \mathbf{b} \sqrt{s}: 1500 \mathrm{GeV}, \mathbf{c} \sqrt{s}: 3000 \mathrm{GeV}$ ), considering $X=10^{-6}$. The red line indicates the innermost tracker layer distance at $165 \mathrm{~mm}$ from the center

results. We have also shown the scalar mass eigenstates and interactions, necessary to our calculations.

Out of the exotic particles involved in the diagrams for the cross-section, the sterile neutrino gives a constant contribution to the final result, regardless of its mass. Its coupling constant $\left(X=F_{1 S}^{v} v_{1} / \Lambda\right.$ ) to the charged fermions and scalars does have an influence on the final results, but still is a small contribution when compared to the one given by the dia-

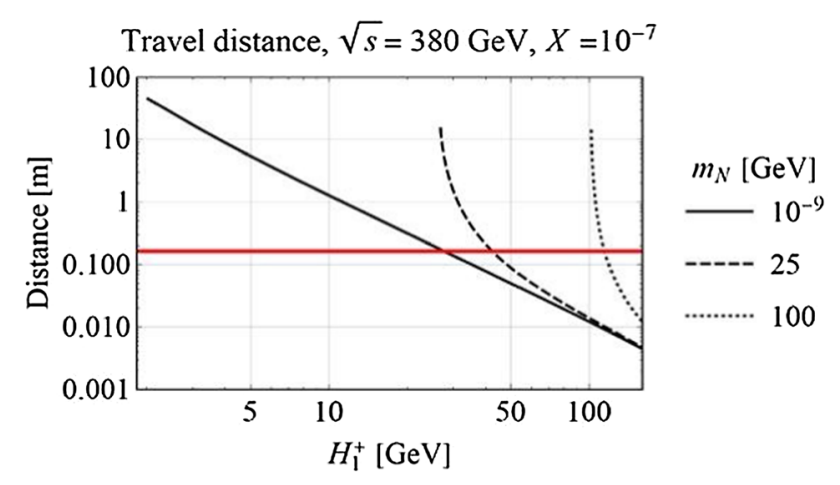

(a)

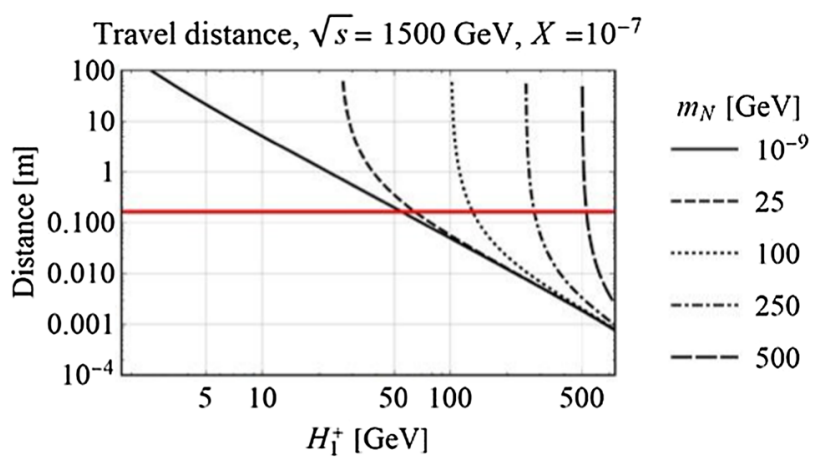

(b)

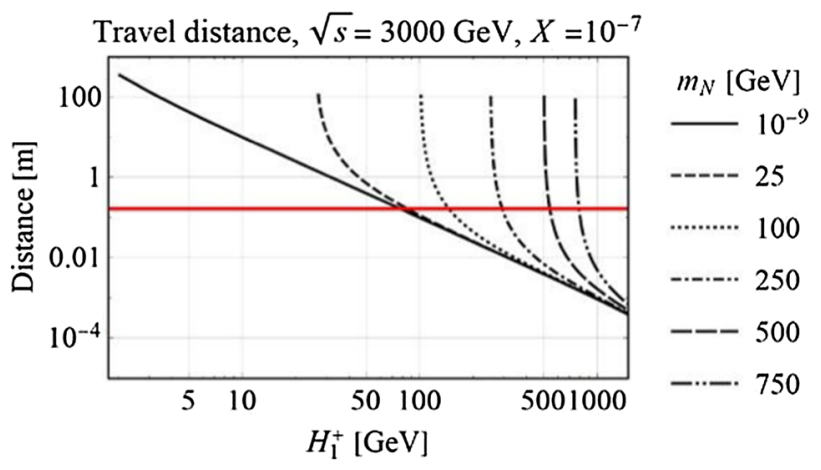

(c)

Fig. $6 H_{1}^{+}$travel distance. The graphs show the total travel distance of $H_{1}^{+}$for different values of $m_{N}$ and energy stages of CLIC $(\mathbf{a} \sqrt{s}: 380$ $\mathrm{GeV}, \mathbf{b} \sqrt{s}: 1500 \mathrm{GeV}, \mathbf{c} \sqrt{s}: 3000 \mathrm{GeV}$ ), considering $X=10^{-7}$. The red line indicates the innermost tracker layer distance at $165 \mathrm{~mm}$ from the center

gram with the photon. However, a parameter that noticeably changes the cross-section values is the charged scalar mass, leading to a difference of orders of magnitude when comparing the lowest and highest possible cross-section values. In the end, the expected number of events depend heavily on the charged scalar mass, ranging from $10^{6}$ all the way down to 250 . Therefore, this mass value can make the difference 
in between an observable and a non-observable production of these exotic particles.

In the case of $\sqrt{s}=380 \mathrm{GeV}$ and large $X$, one may see a noticeable increase in $e \bar{e}+$ missing energy events, allowing for an indirect detection. However, given the small cross section of $e \bar{e} \rightarrow e \bar{e} N \bar{N}$ for $\sqrt{s}=1500$ and $3000 \mathrm{GeV}$ (or $\sqrt{s}=380 \mathrm{GeV}$ and small $X$ ), the fact that its signal would be overwhelmed by the SM background from the $e \bar{e} \rightarrow e \bar{e} v_{e} \bar{v}_{e}$ process in these cases, and the possibility of the charged scalar leaving measurable tracks in the detector, we expect this particle to be detected directly in these situations. Such detection however would not allow us to extract much information about the exotic neutrinos of the model, given that their contribution to the total cross-section can be very small and is independent of its mass. Also, the lifetime of $\mathrm{H}_{1}^{+}$ depends on $m_{N}$ and $X$, therefore, knowing the lifetime of the scalar would still not allow us to figure out the values of these parameters.

Acknowledgements G. De Conto would like to thank Coordenação de Aperfeiçoamento de Pessoal de Nível Superior (CAPES) for financial support. J. M. thanks Cátedras CONACYT project 1753.

Data Availability Statement This manuscript has no associated data or the data will not be deposited. [Authors' comment: The calculations performed require no data beyond those shown or referenced in the text. Our results are all presented either numerically, written or in graphical form in this work].

Open Access This article is licensed under a Creative Commons Attribution 4.0 International License, which permits use, sharing, adaptation, distribution and reproduction in any medium or format, as long as you give appropriate credit to the original author(s) and the source, provide a link to the Creative Commons licence, and indicate if changes were made. The images or other third party material in this article are included in the article's Creative Commons licence, unless indicated otherwise in a credit line to the material. If material is not included in the article's Creative Commons licence and your intended use is not permitted by statutory regulation or exceeds the permitted use, you will need to obtain permission directly from the copyright holder. To view a copy of this licence, visit http://creativecomm ons.org/licenses/by/4.0/.

Funded by SCOAP ${ }^{3}$.

\section{Appendix A: Diagonalization solutions for the $3 \times 3$ CP-even mass matrix}

In this appendix we show all the solutions for the $3 \times 3 \mathrm{CP}-$ even mass matrix discussed in Sect. 5, except the ones where $v_{1}=v_{2}=0$. In the first subsection we consider the vacuum stability condition from Eq. 8, and in the second the condition from Eq. 9. All solutions are presented in the same format: first we show the relations that the parameters must obey, then the diagonalized matrix with the masses squared, and finally the orthogonal diagonalization matrix.
7.1 Using $v_{2}=-v_{1}$,

$$
\begin{aligned}
& \mu_{\zeta}^{2}=\frac{1}{2}\left(-b_{1} v_{S M}^{2}-4 v_{1}^{2}\left(c_{1}+c_{2}\right)+\mu_{12}^{2}\right), \text { and } \\
& \mu_{S M}=-a_{4} v_{S M}^{2}-b_{1} v_{1}^{2} .
\end{aligned}
$$

- Solution $\quad 1: \quad\left\{a_{4} \rightarrow \frac{2 v_{1}^{2}\left(c_{1}+c_{2}\right)}{v_{S M}^{2}}, b_{1} \rightarrow 0, \sin \theta \rightarrow\right.$ $\left.-\sqrt{1-\cos \theta^{2}}, \mu_{12}^{2} \rightarrow 4 v_{1}^{2}\left(c_{1}+c_{2}\right)\right\}$

$$
\begin{aligned}
& \operatorname{diag}\left(m_{H}^{2}, m_{H_{1}^{0}}^{2}, m_{H_{2}^{0}}^{2}\right) \\
& =\left(\begin{array}{ccc}
4 v_{1}^{2}\left(c_{1}+c_{2}\right) & 0 & 0 \\
0 & 4 v_{1}^{2}\left(c_{1}+c_{2}\right) & 0 \\
0 & 0 & 4 v_{1}^{2}\left(c_{1}+c_{2}\right)
\end{array}\right) \\
& R_{E 2}=\left(\begin{array}{ccc}
1 & 0 & 0 \\
0 & \cos \theta & -\sqrt{1-\cos \theta^{2}} \\
0 \sqrt{1-\cos \theta^{2}} & \cos \theta
\end{array}\right)
\end{aligned}
$$

- Solution 2: $\quad\left\{a_{4} \rightarrow \frac{2 v_{1}^{2}\left(c_{1}+c_{2}\right)}{v_{S M}^{2}}, b_{1} \rightarrow 0, \sin \theta \rightarrow\right.$ $\left.\sqrt{1-\cos \theta^{2}}, \mu_{12}^{2} \rightarrow 4 v_{1}^{2}\left(c_{1}+c_{2}\right)\right\}$

$$
\begin{aligned}
& \operatorname{diag}\left(m_{H}^{2}, m_{H_{1}^{0}}^{2}, m_{H_{2}^{0}}^{2}\right) \\
& =\left(\begin{array}{ccc}
4 v_{1}^{2}\left(c_{1}+c_{2}\right) & 0 & 0 \\
0 & 4 v_{1}^{2}\left(c_{1}+c_{2}\right) & 0 \\
0 & 0 & 4 v_{1}^{2}\left(c_{1}+c_{2}\right)
\end{array}\right) \\
& R_{E 2}=\left(\begin{array}{ccc}
1 & 0 & 0 \\
0 & \cos \theta & \sqrt{1-\cos \theta^{2}} \\
0-\sqrt{1-\cos \theta^{2}} & \cos \theta
\end{array}\right)
\end{aligned}
$$

- Solution 3: $\left\{b_{1} \rightarrow 0, \cos \theta \rightarrow-\frac{1}{\sqrt{2}}, \sin \theta \rightarrow-\frac{1}{\sqrt{2}}\right.$, $\left.\mu_{12}^{2} \rightarrow 2 a_{4} v_{S M}^{2}\right\}$

$$
\begin{aligned}
& \operatorname{diag}\left(m_{H}^{2}, m_{H_{1}^{0}}^{2}, m_{H_{2}^{0}}^{2}\right) \\
& =\left(\begin{array}{ccc}
2 a_{4} v_{S M}^{2} & 0 & 0 \\
0 & 4 v_{1}^{2}\left(c_{1}+c_{2}\right) & 0 \\
0 & 0 & 2 a_{4} v_{S M}^{2}
\end{array}\right) \\
& R_{E 2}=\left(\begin{array}{ccc}
1 & 0 & 0 \\
0 & -\frac{1}{\sqrt{2}} & -\frac{1}{\sqrt{2}} \\
0 & \frac{1}{\sqrt{2}} & -\frac{1}{\sqrt{2}}
\end{array}\right)
\end{aligned}
$$

- Solution 4: $\quad\left\{b_{1} \rightarrow 0, \cos \theta \rightarrow \frac{1}{\sqrt{2}}, \sin \theta \rightarrow-\frac{1}{\sqrt{2}}\right.$, $\left.\mu_{12}^{2} \rightarrow 2 a_{4} v_{S M}^{2}\right\}$ 


$$
\begin{aligned}
& \operatorname{diag}\left(m_{H}^{2}, m_{H_{1}^{0}}^{2}, m_{H_{2}^{0}}^{2}\right) \\
& =\left(\begin{array}{ccc}
2 a_{4} v_{S M}^{2} & 0 & 0 \\
0 & 2 a_{4} v_{S M}^{2} & 0 \\
0 & 0 & 4 v_{1}^{2}\left(c_{1}+c_{2}\right)
\end{array}\right) \\
& R_{E 2}=\left(\begin{array}{ccc}
1 & 0 & 0 \\
0 & \frac{1}{\sqrt{2}}-\frac{1}{\sqrt{2}} \\
0 & \frac{1}{\sqrt{2}} & \frac{1}{\sqrt{2}}
\end{array}\right)
\end{aligned}
$$

- Solution 5: $\quad\left\{b_{1} \rightarrow 0, \cos \theta \rightarrow-\frac{1}{\sqrt{2}}, \sin \theta \rightarrow \frac{1}{\sqrt{2}}\right.$, $\left.\mu_{12}^{2} \rightarrow 2 a_{4} v_{S M}^{2}\right\}$

$$
\begin{aligned}
& \operatorname{diag}\left(m_{H}^{2}, m_{H_{1}^{0}}^{2}, m_{H_{2}^{0}}^{2}\right) \\
& =\left(\begin{array}{ccc}
2 a_{4} v_{S M}^{2} & 0 & 0 \\
0 & 2 a_{4} v_{S M}^{2} & 0 \\
0 & 0 & 4 v_{1}^{2}\left(c_{1}+c_{2}\right)
\end{array}\right) \\
& R_{E 2}=\left(\begin{array}{ccc}
1 & 0 & 0 \\
0-\frac{1}{\sqrt{2}} & \frac{1}{\sqrt{2}} \\
0-\frac{1}{\sqrt{2}} & -\frac{1}{\sqrt{2}}
\end{array}\right)
\end{aligned}
$$

- Solution 6: $\quad\left\{b_{1} \rightarrow 0, \cos \theta \rightarrow \frac{1}{\sqrt{2}}, \sin \theta \rightarrow \frac{1}{\sqrt{2}}\right.$, $\left.\mu_{12}^{2} \rightarrow 2 a_{4} v_{S M}^{2}\right\}$

$$
\begin{aligned}
& \operatorname{diag}\left(m_{H}^{2}, m_{H_{1}^{0}}^{2}, m_{H_{2}^{0}}^{2}\right) \\
& =\left(\begin{array}{ccc}
2 a_{4} v_{S M}^{2} & 0 & 0 \\
0 & 4 v_{1}^{2}\left(c_{1}+c_{2}\right) & 0 \\
0 & 0 & 2 a_{4} v_{S M}^{2}
\end{array}\right) \\
& R_{E 2}=\left(\begin{array}{ccc}
1 & 0 & 0 \\
0 & \frac{1}{\sqrt{2}} & \frac{1}{\sqrt{2}} \\
0 & -\frac{1}{\sqrt{2}} & \frac{1}{\sqrt{2}}
\end{array}\right)
\end{aligned}
$$

- Solution 7: $\left\{a_{4} \rightarrow \frac{2 v_{1}^{2}\left(c_{1}+c_{2}\right)}{v_{S M}^{2}}, b_{1} \rightarrow 0, \cos \theta \rightarrow-\frac{1}{\sqrt{2}}\right.$,

$$
\begin{aligned}
& \left.\sin \theta \rightarrow-\frac{1}{\sqrt{2}}, \mu_{12}^{2} \rightarrow 4 v_{1}^{2}\left(c_{1}+c_{2}\right)\right\} \\
& \operatorname{diag}\left(m_{H}^{2}, m_{H_{1}^{0}}^{2}, m_{H_{2}^{0}}^{2}\right) \\
& =\left(\begin{array}{ccc}
4 v_{1}^{2}\left(c_{1}+c_{2}\right) & 0 & 0 \\
0 & 4 v_{1}^{2}\left(c_{1}+c_{2}\right) & 0 \\
0 & 0 & 4 v_{1}^{2}\left(c_{1}+c_{2}\right)
\end{array}\right) \\
& R_{E 2}=\left(\begin{array}{ccc}
1 & 0 & 0 \\
0 & -\frac{1}{\sqrt{2}} & -\frac{1}{\sqrt{2}} \\
0 & \frac{1}{\sqrt{2}} & -\frac{1}{\sqrt{2}}
\end{array}\right)
\end{aligned}
$$

- Solution 8: $\left\{a_{4} \rightarrow \frac{2 v_{1}^{2}\left(c_{1}+c_{2}\right)}{v_{S M}^{2}}, b_{1} \rightarrow 0, \cos \theta \rightarrow \frac{1}{\sqrt{2}}\right.$,

$$
\left.\sin \theta \rightarrow-\frac{1}{\sqrt{2}}, \mu_{12}^{2} \rightarrow 4 v_{1}^{2}\left(c_{1}+c_{2}\right)\right\}
$$

$$
\begin{aligned}
& \operatorname{diag}\left(m_{H}^{2}, m_{H_{1}^{0}}^{2}, m_{H_{2}^{0}}^{2}\right) \\
& =\left(\begin{array}{ccc}
4 v_{1}^{2}\left(c_{1}+c_{2}\right) & 0 & 0 \\
0 & 4 v_{1}^{2}\left(c_{1}+c_{2}\right) & 0 \\
0 & 0 & 4 v_{1}^{2}\left(c_{1}+c_{2}\right)
\end{array}\right)
\end{aligned}
$$

$$
R_{E 2}=\left(\begin{array}{ccc}
1 & 0 & 0 \\
0 & \frac{1}{\sqrt{2}} & -\frac{1}{\sqrt{2}} \\
0 & \frac{1}{\sqrt{2}} & \frac{1}{\sqrt{2}}
\end{array}\right)
$$

- Solution 9: $\left\{a_{4} \rightarrow \frac{2 v_{1}^{2}\left(c_{1}+c_{2}\right)}{v_{S M}^{2}}, b_{1} \rightarrow 0, \cos \theta \rightarrow-\frac{1}{\sqrt{2}}\right.$,

$$
\left.\sin \theta \rightarrow \frac{1}{\sqrt{2}}, \mu_{12}^{2} \rightarrow 4 v_{1}^{2}\left(c_{1}+c_{2}\right)\right\}
$$

$$
\begin{aligned}
& \operatorname{diag}\left(m_{H}^{2}, m_{H_{1}^{0}}^{2}, m_{H_{2}^{0}}^{2}\right) \\
& =\left(\begin{array}{ccc}
4 v_{1}^{2}\left(c_{1}+c_{2}\right) & 0 & 0 \\
0 & 4 v_{1}^{2}\left(c_{1}+c_{2}\right) & 0 \\
0 & 0 & 4 v_{1}^{2}\left(c_{1}+c_{2}\right)
\end{array}\right)
\end{aligned}
$$

$$
R_{E 2}=\left(\begin{array}{ccc}
1 & 0 & 0 \\
0 & -\frac{1}{\sqrt{2}} & \frac{1}{\sqrt{2}} \\
0 & -\frac{1}{\sqrt{2}} & -\frac{1}{\sqrt{2}}
\end{array}\right)
$$

- Solution 10: $\left\{a_{4} \rightarrow \frac{2 v_{1}^{2}\left(c_{1}+c_{2}\right)}{v_{S M}^{2}}, b_{1} \rightarrow 0, \cos \theta \rightarrow \frac{1}{\sqrt{2}}\right.$,

$$
\left.\sin \theta \rightarrow \frac{1}{\sqrt{2}}, \mu_{12}^{2} \rightarrow 4 v_{1}^{2}\left(c_{1}+c_{2}\right)\right\}
$$$$
\operatorname{diag}\left(m_{H}^{2}, m_{H_{1}^{0}}^{2}, m_{H_{2}^{0}}^{2}\right)
$$

$$
=\left(\begin{array}{ccc}
4 v_{1}^{2}\left(c_{1}+c_{2}\right) & 0 & 0 \\
0 & 4 v_{1}^{2}\left(c_{1}+c_{2}\right) & 0 \\
0 & 0 & 4 v_{1}^{2}\left(c_{1}+c_{2}\right)
\end{array}\right)
$$

$$
R_{E 2}=\left(\begin{array}{ccc}
1 & 0 & 0 \\
0 & \frac{1}{\sqrt{2}} & \frac{1}{\sqrt{2}} \\
0 & -\frac{1}{\sqrt{2}} & \frac{1}{\sqrt{2}}
\end{array}\right)
$$

7.2 Using $v_{2}=v_{1}$,

$$
\begin{aligned}
& \mu_{\zeta}^{2}=\frac{1}{2}\left(-b_{1} v_{S M}^{2}-4 v_{1}^{2}\left(c_{1}+c_{2}\right)-\mu_{12}^{2}\right) \text { and } \\
& \mu_{S M}=-a_{4} v_{S M}^{2}-b_{1} v_{1}^{2} .
\end{aligned}
$$

- Solution $\quad 1: \quad\left\{a_{4} \rightarrow \frac{2 v_{1}^{2}\left(c_{1}+c_{2}\right)}{v_{S M}^{2}}, b_{1} \rightarrow 0, \sin \theta \rightarrow\right.$ $\left.-\sqrt{1-\cos \theta^{2}}, \mu_{12}^{2} \rightarrow-4 v_{1}^{2}\left(c_{1}+c_{2}\right)\right\}$ $\operatorname{diag}\left(m_{H}^{2}, m_{H_{1}^{0}}^{2}, m_{H_{2}^{0}}^{2}\right)$ 


$$
\begin{array}{rlrl}
= & \left(\begin{array}{ccc}
4 v_{1}^{2}\left(c_{1}+c_{2}\right) & 0 & 0 \\
0 & 4 v_{1}^{2}\left(c_{1}+c_{2}\right) & 0 \\
0 & 0 & 4 v_{1}^{2}\left(c_{1}+c_{2}\right)
\end{array}\right) & =\left(\begin{array}{ccc}
2 a_{4} v_{S M}^{2} & 0 & 0 \\
0 & 4 v_{1}^{2}\left(c_{1}+c_{2}\right) & 0 \\
0 & 0 & 2 a_{4} v_{S M}^{2}
\end{array}\right) \\
R_{E 2}=\left(\begin{array}{ccc}
1 & 0 & 0 \\
0 & \cos \theta & -\sqrt{1-\cos \theta^{2}} \\
0 \sqrt{1-\cos \theta^{2}} & \cos \theta
\end{array}\right) & R_{E 2}=\left(\begin{array}{ccc}
1 & 0 & 0 \\
0-\frac{1}{\sqrt{2}} & \frac{1}{\sqrt{2}} \\
0-\frac{1}{\sqrt{2}} & -\frac{1}{\sqrt{2}}
\end{array}\right)
\end{array}
$$

- Solution $\quad 2: \quad\left\{a_{4} \rightarrow \frac{2 v_{1}^{2}\left(c_{1}+c_{2}\right)}{v_{S M}^{2}}, b_{1} \rightarrow 0, \sin \theta \rightarrow\right.$ $\left.\sqrt{1-\cos \theta^{2}}, \mu_{12}^{2} \rightarrow-4 v_{1}^{2}\left(c_{1}+c_{2}\right)\right\}$

$$
\begin{aligned}
& \operatorname{diag}\left(m_{H}^{2}, m_{H_{1}^{0}}^{2}, m_{H_{2}^{0}}^{2}\right) \\
& =\left(\begin{array}{ccc}
4 v_{1}^{2}\left(c_{1}+c_{2}\right) & 0 & 0 \\
0 & 4 v_{1}^{2}\left(c_{1}+c_{2}\right) & 0 \\
0 & 0 & 4 v_{1}^{2}\left(c_{1}+c_{2}\right)
\end{array}\right) \\
& R_{E 2}=\left(\begin{array}{ccc}
1 & 0 & 0 \\
0 & \cos \theta & \sqrt{1-\cos \theta^{2}} \\
0-\sqrt{1-\cos \theta^{2}} & \cos \theta
\end{array}\right)
\end{aligned}
$$

- Solution 3: $\left\{b_{1} \rightarrow 0, \cos \theta \rightarrow-\frac{1}{\sqrt{2}}, \sin \theta \rightarrow-\frac{1}{\sqrt{2}}\right.$, $\left.\mu_{12}^{2} \rightarrow-2 a_{4} v_{S M}^{2}\right\}$

$$
\begin{aligned}
& \operatorname{diag}\left(m_{H}^{2}, m_{H_{1}^{0}}^{2}, m_{H_{2}^{0}}^{2}\right) \\
& =\left(\begin{array}{ccc}
2 a_{4} v_{S M}^{2} & 0 & 0 \\
0 & 2 a_{4} v_{S M}^{2} & 0 \\
0 & 0 & 4 v_{1}^{2}\left(c_{1}+c_{2}\right)
\end{array}\right) \\
& R_{E 2}=\left(\begin{array}{ccc}
1 & 0 & 0 \\
0 & -\frac{1}{\sqrt{2}} & -\frac{1}{\sqrt{2}} \\
0 & \frac{1}{\sqrt{2}} & -\frac{1}{\sqrt{2}}
\end{array}\right)
\end{aligned}
$$

- Solution 4: $\left\{b_{1} \rightarrow 0, \cos \theta \rightarrow \frac{1}{\sqrt{2}}, \sin \theta \rightarrow-\frac{1}{\sqrt{2}}, \mu_{12}^{2}\right.$ $\left.\rightarrow-2 a_{4} v_{S M}^{2}\right\}$

$$
\begin{aligned}
& \operatorname{diag}\left(m_{H}^{2}, m_{H_{1}^{0}}^{2}, m_{H_{2}^{0}}^{2}\right) \\
& =\left(\begin{array}{ccc}
2 a_{4} v_{S M}^{2} & 0 & 0 \\
0 & 4 v_{1}^{2}\left(c_{1}+c_{2}\right) & 0 \\
0 & 0 & 2 a_{4} v_{S M}^{2}
\end{array}\right) \\
& R_{E 2}=\left(\begin{array}{ccc}
1 & 0 & 0 \\
0 & \frac{1}{\sqrt{2}}-\frac{1}{\sqrt{2}} \\
0 & \frac{1}{\sqrt{2}} & \frac{1}{\sqrt{2}}
\end{array}\right)
\end{aligned}
$$

- Solution 5: $\left\{b_{1} \rightarrow 0, \cos \theta \rightarrow-\frac{1}{\sqrt{2}}, \sin \theta \rightarrow \frac{1}{\sqrt{2}}\right.$, $\left.\mu_{12}^{2} \rightarrow-2 a_{4} v_{S M}^{2}\right\}$ $\operatorname{diag}\left(m_{H}^{2}, m_{H_{1}^{0}}^{2}, m_{H_{2}^{0}}^{2}\right)$
- Solution 6: $\left\{b_{1} \rightarrow 0, \cos \theta \rightarrow \frac{1}{\sqrt{2}}, \sin \theta \rightarrow \frac{1}{\sqrt{2}}\right.$, $\left.\mu_{12}^{2} \rightarrow-2 a_{4} v_{S M}^{2}\right\}$

$$
\begin{aligned}
& \operatorname{diag}\left(m_{H}^{2}, m_{H_{1}^{0}}^{2}, m_{H_{2}^{0}}^{2}\right) \\
& =\left(\begin{array}{ccc}
2 a_{4} v_{S M}^{2} & 0 & 0 \\
0 & 2 a_{4} v_{S M}^{2} & 0 \\
0 & 0 & 4 v_{1}^{2}\left(c_{1}+c_{2}\right)
\end{array}\right) \\
& R_{E 2}=\left(\begin{array}{ccc}
1 & 0 & 0 \\
0 & \frac{1}{\sqrt{2}} & \frac{1}{\sqrt{2}} \\
0-\frac{1}{\sqrt{2}} & \frac{1}{\sqrt{2}}
\end{array}\right)
\end{aligned}
$$

- Solution 7: $\left\{a_{4} \rightarrow \frac{2 v_{1}^{2}\left(c_{1}+c_{2}\right)}{v_{S M}^{2}}, b_{1} \rightarrow 0, \cos \theta \rightarrow-\frac{1}{\sqrt{2}}\right.$,

$$
\left.\sin \theta \rightarrow-\frac{1}{\sqrt{2}}, \mu_{12}^{2} \rightarrow-4 v_{1}^{2}\left(c_{1}+c_{2}\right)\right\}
$$

$$
\begin{aligned}
& \operatorname{diag}\left(m_{H}^{2}, m_{H_{1}^{0}}^{2}, m_{H_{2}^{0}}^{2}\right) \\
& =\left(\begin{array}{ccc}
4 v_{1}^{2}\left(c_{1}+c_{2}\right) & 0 & 0 \\
0 & 4 v_{1}^{2}\left(c_{1}+c_{2}\right) & 0 \\
0 & 0 & 4 v_{1}^{2}\left(c_{1}+c_{2}\right)
\end{array}\right) \\
& R_{E 2}=\left(\begin{array}{ccc}
1 & 0 & 0 \\
0 & -\frac{1}{\sqrt{2}} & -\frac{1}{\sqrt{2}} \\
0 & \frac{1}{\sqrt{2}} & -\frac{1}{\sqrt{2}}
\end{array}\right)
\end{aligned}
$$

- Solution 8: $\left\{a_{4} \rightarrow \frac{2 v_{1}^{2}\left(c_{1}+c_{2}\right)}{v_{S M}^{2}}, b_{1} \rightarrow 0, \cos \theta \rightarrow \frac{1}{\sqrt{2}}\right.$,

$$
\left.\sin \theta \rightarrow-\frac{1}{\sqrt{2}}, \mu_{12}^{2} \rightarrow-4 v_{1}^{2}\left(c_{1}+c_{2}\right)\right\}
$$

$$
\begin{aligned}
& \operatorname{diag}\left(m_{H}^{2}, m_{H_{1}^{0}}^{2}, m_{H_{2}^{0}}^{2}\right) \\
& =\left(\begin{array}{ccc}
4 v_{1}^{2}\left(c_{1}+c_{2}\right) & 0 & 0 \\
0 & 4 v_{1}^{2}\left(c_{1}+c_{2}\right) & 0 \\
0 & 0 & 4 v_{1}^{2}\left(c_{1}+c_{2}\right)
\end{array}\right)
\end{aligned}
$$$$
R_{E 2}=\left(\begin{array}{ccc}
1 & 0 & 0 \\
0 & \frac{1}{\sqrt{2}} & -\frac{1}{\sqrt{2}} \\
0 & \frac{1}{\sqrt{2}} & \frac{1}{\sqrt{2}}
\end{array}\right)
$$ 
- Solution 9: $\left\{a_{4} \rightarrow \frac{2 v_{1}^{2}\left(c_{1}+c_{2}\right)}{v_{S M}^{2}}, b_{1} \rightarrow 0, \cos \theta \rightarrow-\frac{1}{\sqrt{2}}\right.$, $\left.\sin \theta \rightarrow \frac{1}{\sqrt{2}}, \mu_{12}^{2} \rightarrow-4 v_{1}^{2}\left(c_{1}+c_{2}\right)\right\}$

$$
\operatorname{diag}\left(m_{H}^{2}, m_{H_{1}^{0}}^{2}, m_{H_{2}^{0}}^{2}\right)
$$$$
=\left(\begin{array}{ccc}
4 v_{1}^{2}\left(c_{1}+c_{2}\right) & 0 & 0 \\
0 & 4 v_{1}^{2}\left(c_{1}+c_{2}\right) & 0 \\
0 & 0 & 4 v_{1}^{2}\left(c_{1}+c_{2}\right)
\end{array}\right)
$$$$
R_{E 2}=\left(\begin{array}{ccc}
1 & 0 & 0 \\
0 & -\frac{1}{\sqrt{2}} & \frac{1}{\sqrt{2}} \\
0 & -\frac{1}{\sqrt{2}} & -\frac{1}{\sqrt{2}}
\end{array}\right)
$$

- Solution 10: $\left\{a_{4} \rightarrow \frac{2 v_{1}^{2}\left(c_{1}+c_{2}\right)}{v_{S M}^{2}}, b_{1} \rightarrow 0, \cos \theta \rightarrow \frac{1}{\sqrt{2}}\right.$, $\left.\sin \theta \rightarrow \frac{1}{\sqrt{2}}, \mu_{12}^{2} \rightarrow-4 v_{1}^{2}\left(c_{1}+c_{2}\right)\right\}$

$$
\begin{aligned}
& \operatorname{diag}\left(m_{H}^{2}, m_{H_{1}^{0}}^{2}, m_{H_{2}^{0}}^{2}\right) \\
& =\left(\begin{array}{ccc}
4 v_{1}^{2}\left(c_{1}+c_{2}\right) & 0 & 0 \\
0 & 4 v_{1}^{2}\left(c_{1}+c_{2}\right) & 0 \\
0 & 0 & 4 v_{1}^{2}\left(c_{1}+c_{2}\right)
\end{array}\right) \\
& R_{E 2}=\left(\begin{array}{ccc}
1 & 0 & 0 \\
0 & \frac{1}{\sqrt{2}} & \frac{1}{\sqrt{2}} \\
0-\frac{1}{\sqrt{2}} & \frac{1}{\sqrt{2}}
\end{array}\right)
\end{aligned}
$$

\section{References}

1. L. Bergstrom, Non-baryonic dark matter: observational evidence and detection methods. Rep. Prog. Phys. 63, 793 (2000). arXiv:hep-ph/0002126 [SPIRES]

2. G. Bertone, D. Hooper, J. Silk, Particle dark matter: evidence, candidates and constraints. Phys. Rep. 405, 279 (2005). arXiv:hep-ph/0404175 [SPIRES]

3. J. Carr, G. Lamanna, J. Lavalle, Indirect detection of dark matter. Rep. Prog. Phys. 69, 2475 (2006). [SPIRES]

4. M. Taoso, G. Bertone, A. Masiero, Dark matter candidates: a tenpoint test. JCAP 03, 022 (2008). arXiv:0711.4996 [SPIRES]

5. S.L. Dubovsky, P.G. Tinyakov, I.I. Tkachev, Massive graviton as a testable cold dark matter candidate. Phys. Rev. Lett. 94, 181102 (2005). arXiv:hep-th/0411158 [SPIRES]

6. R. Holman, G. Lazarides, Q. Shafi, Axions and the dark matter of the universe. Phys. Rev. D 27, 995 (1983). [SPIRES]

7. S. Dodelson, L.M. Widrow, Sterile-neutrinos as dark matter. Phys. Rev. Lett. 72, 17-20 (1994)

8. H. Pagels, J.R. Primack, Supersymmetry, cosmology and new physics at TeV energies. Phys. Rev. Lett. 48, 223 (1982). [SPIRES]

9. H.E. Haber, G.L. Kane, The search for supersymmetry: probing physics beyond the standard model. Phys. Rep. 117, 75 (1985). [SPIRES]
10. L. Covi, J.E. Kim, L. Roszkowski, Axinos as cold dark matter. Phys. Rev. Lett. 82, 4180 (1999). arXiv:hep-ph/9905212 [SPIRES]

11. A. Kusenko, M.E. Shaposhnikov, Supersymmetric Q-balls as dark matter. Phys. Lett. B 418, 46 (1998). arXiv:hep-ph/9709492 [SPIRES]

12. D.J.H. Chung, E.W. Kolb, A. Riotto, Superheavy dark matter. Phys. Rev. D 59, 023501 (1999). arXiv:hep-ph/9802238 [SPIRES]

13. V. Kuzmin, I. Tkachev, Ultra-high energy cosmic rays, superheavy long-living particles and matter creation after inflation. JETP Lett. 68, 271 (1998). arXiv:hep-ph/9802304 [SPIRES]

14. A. Boyarsky, M. Drewes, T. Lasserre, S. Mertens, O. Ruchayskiy, Prog. Part. Nucl. Phys. 104, 1 (2019). https://doi.org/10.1016/j. ppnp.2018.07.004. arXiv:1807.07938 [hep-ph]

15. T. Asaka, S. Blanchet, M. Shaposhnikov, The vMSM, dark matter and neutrino masses. Phys. Lett. B 631, 151 (2005). arXiv:hep-ph/0503065 [SPIRES]

16. G.L. Fogli, E. Lisi, A. Marrone, A. Palazzo, Global analysis of three-flavor neutrino masses and mixings. Prog. Part. Nucl. Phys. 57, 742 (2006). arXiv:hep-ph/0506083 [SPIRES]

17. A. Strumia, F. Vissani, Neutrino masses and mixings. arXiv:hep-ph/0606054 [SPIRES]

18. C. Giunti, Theory and phenomenology of neutrino mixing. Nucl. Phys. Proc. Suppl. 169, 309 (2007). arXiv:hep-ph/0611125 [SPIRES]

19. A.D. Dolgov, Baryogenesis, 30 years after. arXiv:hep-ph/9707419 [SPIRES]

20. A. Riotto, Theories of baryogenesis. arXiv:hep-ph/9807454 [SPIRES]

21. A.G. Dias, A.C.B. Machado, C.C. Nishi, Phys. Rev. D 86, 093005 (2012). https://doi.org/10.1103/PhysRevD.86.093005. arXiv:1206.6362 [hep-ph]

22. E.C.F.S. Fortes, A.C.B. Machado, J. Montaño, V. Pleitez, Phys. Lett. B 803, 135289 (2020). https://doi.org/10.1016/j.physletb. 2020.135289

23. J. Kubo, H. Okada, F. Sakamaki, Phys. Rev. D 70, 036007 (2004). https://doi.org/10.1103/PhysRevD.70.036007. arXiv:hep-ph/0402089

24. E. Barradas-Guevara, O. Félix-Beltrán, E. Rodríguez-Jáuregui, Phys. Rev. D 90(9), 095001 (2014). https://doi.org/10.1103/ PhysRevD.90.095001. arXiv:1402.2244 [hep-ph]

25. D. Das, U.K. Dey, Phys. Rev. D 89(9), 095025 (2014) https://doi.org/10.1103/PhysRevD.91.039905. https://doi.org/10. 1103/PhysRevD.89.095025. arXiv:1404.2491 [hep-ph] [Erratum: Phys. Rev. D 91, no. 3, 039905 (2015)]

26. F. González Canales, A. Mondragón, M. Mondragón, U.J. Saldaña Salazar, L. Velasco-Sevilla, Phys. Rev. D 88, 096004 (2013). https://doi.org/10.1103/PhysRevD.88.096004. arXiv:1304.6644 [hep-ph]

27. F. Gonzalez Canales, A. Mondragon, M. Mondragon, Fortsch. Phys. 61, 546 (2013). https://doi.org/10.1002/prop.201200121. arXiv:1205.4755 [hep-ph]

28. A. Riotto, M. Trodden, Annu. Rev. Nucl. Part. Sci. 49, 35 (1999)

29. D.E. Morrissey, M.J. Ramsey-Musolf, New J. Phys. 14, 125003 (2012)

30. M. Dine, A. Kusenko, Rev. Mod. Phys. 76(1), 1 (2004)

31. A.C.B. Machado, J.C. Montero, V. Pleitez, Phys. Rev. D 88(11), 113002 (2013). https://doi.org/10.1103/PhysRevD.88. 113002. arXiv:1305.1921 [hep-ph]

32. G. Castelo Branco, L. Lavoura, J.P. Silva, CP Violation (Claredon Press, Oxford, 1999)

33. P. Roloff et al. [CLIC and CLICdp Collaborations], arXiv:1812.07986 [hep-ex]

34. A. Robson et al., arXiv:1812.07987 [physics.acc-ph]

35. H. Ishimori, T. Kobayashi, H. Ohki, Y. Shimizu, H. Okada, M. Tanimoto, Prog. Theor. Phys. Suppl. 183, 1 (2010). https://doi.org/ 10.1143/PTPS.183.1. arXiv:1003.3552 [hep-th] 
36. G. De Conto, A.C.B. Machado, J.P.B.C. de Melo, Phys. Lett. B 784, 255 (2018). https://doi.org/10.1016/j.physletb.2018.07.056. arXiv:1711.06718 [hep-ph]

37. M. Tanabashi et al. (Particle Data Group), Phys. Rev. D 98, 030001 (2018)
38. Physics and Detectors at CLIC: CLIC Conceptual Design Report, edited by L. Linssen, A. Miyamoto, M. Stanitzki, H. Weerts, CERN-2012-003. arXiv:1202.5940 